\title{
MULTI-PEAK SOLUTIONS FOR MAGNETIC NLS EQUATIONS WITHOUT NON-DEGENERACY CONDITIONS *,**
}

\author{
Silvia Cingolani ${ }^{1}$, Louis Jeanjean ${ }^{2}$ And Simone SeCChi $^{3}$
}

Abstract. In this work we consider the magnetic NLS equation

$$
\left(\frac{\hbar}{i} \nabla-A(x)\right)^{2} u+V(x) u-f\left(|u|^{2}\right) u=0 \quad \text { in } \mathbb{R}^{N}
$$

where $N \geq 3, A: \mathbb{R}^{N} \rightarrow \mathbb{R}^{N}$ is a magnetic potential, possibly unbounded, $V: \mathbb{R}^{N} \rightarrow \mathbb{R}$ is a multi-well electric potential, which can vanish somewhere, $f$ is a subcritical nonlinear term. We prove the existence of a semiclassical multi-peak solution $u: \mathbb{R}^{N} \rightarrow \mathbb{C}$ to $(0.1)$, under conditions on the nonlinearity which are nearly optimal.

Mathematics Subject Classification. 35J20, 35J60.

Received October 16, 2007. Revised March 13, 2008.

Published online August 20, 2008.

\section{INTRODUCTION}

We study the existence of a standing wave solution $\psi(x, t)=\exp (-i E t / \hbar) u(x), E \in \mathbb{R}, u: \mathbb{R}^{N} \rightarrow \mathbb{C}$ to the time-dependent nonlinear Schrödinger equation in the presence of an external electromagnetic field

$$
i \hbar \frac{\partial \psi}{\partial t}=\left(\frac{\hbar}{i} \nabla-A(x)\right)^{2} \psi+V(x) \psi-f\left(|\psi|^{2}\right) \psi, \quad(t, x) \in \mathbb{R} \times \mathbb{R}^{N} .
$$

Here $\hbar$ is the Planck's constant, $i$ the imaginary unit, $A: \mathbb{R}^{N} \rightarrow \mathbb{R}^{N}$ denotes a magnetic potential and $V: \mathbb{R}^{N} \rightarrow$ $\mathbb{R}$ an electric potential. This leads us to solve the complex semilinear elliptic equation

$$
\left(\frac{\hbar}{i} \nabla-A(x)\right)^{2} u+(V(x)-E) u-f\left(|u|^{2}\right) u=0, \quad x \in \mathbb{R}^{N} .
$$

Keywords and phrases. Nonlinear Schrödinger equations, magnetic fields, multi-peaks.

* The first author is supported by MIUR, national project Variational and topological methods in the study of nonlinear phenomena (PRIN 2005).

** The third author is supported by MIUR, national project Variational methods and nonlinear differential equations.

${ }^{1}$ Dipartimento di Matematica, Politecnico di Bari, via Orabona 4, 70125 Bari, Italy. s.cingolani@poliba.it

2 Équipe de Mathématiques (UMR CNRS 6623), 16 Route de Gray, 25030 Besançon, France. louis.jeanjean@univ-fcomte.fr

${ }^{3}$ Dipartimento di Matematica ed Applicazioni, Università di Milano-Bicocca, via Cozzi 53, 20125 Milano, Italy.

Simone.Secchi@unimib.it 
In the work we are interested to seek for solutions of (1.2), which exist for small value of the Planck constant $\hbar>0$. From a mathematical point of view, the transition from quantum to classical mechanics can be formally performed by letting $\hbar \rightarrow 0$, and such solutions, which are usually referred semiclassical bound states, have an important physical meaning.

For simplicity and without loss of generality, we set $\hbar=\varepsilon$ and we shift $E$ to 0 . Set $v(x)=u(\varepsilon x), A_{\varepsilon}(x)=$ $A(\varepsilon x)$ and $V_{\varepsilon}(x)=V(\varepsilon x)$, equation (1.2) is equivalent to

$$
\left(\frac{1}{i} \nabla-A_{\varepsilon}(x)\right)^{2} v+V_{\varepsilon}(x) v-f\left(|v|^{2}\right) v=0, \quad x \in \mathbb{R}^{N} .
$$

In recent years a considerable amount of work has been devoted to investigating standing wave solutions of (1.1) in the case $A=0$. Among others we refer to $[1,2,7,9-11,13,17,18,24-26,28,30,34,36,38,40,45]$. On the contrary still relatively few papers deal with the case $A \neq 0$, namely when a magnetic field is present. The first result on magnetic NLS equations is due to Esteban and Lions. In [27], they prove the existence of standing waves to (1.1) by a constrained minimization approach, in the case $V(x)=1$, for $\hbar>0$ fixed and for special classes of magnetic fields. Successively in [35], Kurata showed that equation (1.3) admits, under some assumptions linking the magnetic and electric potentials, a least energy solution and that this solution concentrates near the set of global minima of $V$, as $\hbar \rightarrow 0$. It is also proved that the magnetic potential $A$ only contributes to the phase factor of the solution of (1.3) for $\hbar>0$ sufficiently small. A multiplicity result for solutions of (1.3) near global minima of $V$ has been obtained in [16] using topological arguments. A solution that concentrates as $\hbar \rightarrow 0$ around an arbitrary non-degenerate critical point of $V$ has been obtained in [19] but only for bounded magnetic potentials. Subsequently this result was extended in [20] to cover also degenerate, but topologically non trivial, critical points of $V$ and to handle general unbounded magnetic potentials $A$. If $A$ and $V$ are periodic functions, the existence of various type of solutions for $\hbar>0$ fixed has been proved in [3] by applying minimax arguments. We also mention the works [4,15] that deal with critical nonlinearities.

Concerning multi-well electric potentials, an existence result of multi-peak solutions to the magnetic NLS equation (1.3) is established by Bartsch et al. in [5], assuming that the function $f$ is increasing on $(0,+\infty)$ and satisfies the Ambrosetti-Rabinowitz's superquadraticity condition. Also, in [5], an isolatedness condition on the least energy level of the limiting equation

$$
-\Delta u+b u-f\left(|u|^{2}\right) u=0, \quad u \in H^{1}\left(\mathbb{R}^{N}, \mathbb{C}\right)
$$

is required to hold for any $b>0$.

In the present paper we prove an existence result of multi-peak solutions to (1.3), under conditions on $f$, that we believe to be nearly optimal. In particular we drop the isolatedness condition, required in [5] and we cover the case of nonlinearities, which are not monotone.

Precisely, the following conditions will be retained.

(A1): $A: \mathbb{R}^{N} \rightarrow \mathbb{R}^{N}$ is of class $C^{1}$.

(V1): $V \in \mathrm{C}^{0}\left(\mathbb{R}^{N}, \mathbb{R}\right), 0 \leq V_{0}=\inf _{x \in \mathbb{R}^{N}} V(x)$ and $\liminf _{|x| \rightarrow \infty} V(x)>0$.

(V2): There are bounded disjoint open sets $O^{1}, \ldots, O^{k}$ such that

$$
0<m_{i}=\inf _{x \in O^{i}} V(x)<\min _{x \in \partial O^{i}} V(x)
$$

for $i=1, \ldots, k$.

For each $i \in\{1, \ldots, k\}$, we define

$$
\mathcal{M}^{i}=\left\{x \in O^{i} \mid V(x)=m_{i}\right\}
$$

and we set $Z=\left\{x \in \mathbb{R}^{N} \mid V(x)=0\right\}$ and $m=\min _{i \in\{1, \ldots, k\}} m_{i}$. 
On the nonlinearity $f$, we require that

(fo): $f:(0,+\infty) \rightarrow \mathbb{R}$ is continuous;

(f1): $\lim _{t \rightarrow 0^{+}} f(t)=0$ if $Z=\emptyset$, and $\limsup _{t \rightarrow 0^{+}} f\left(t^{2}\right) / t^{\mu}<+\infty$ for some $\mu>0$ if $Z \neq \emptyset$;

(f2): there exists some $0<p<\frac{4}{N-2}, N \geq 3$ such that $\lim \sup _{t \rightarrow+\infty} f\left(t^{2}\right) / t^{p}<+\infty$;

(f3): there exists $T>0$ such that $\frac{1}{2} \hat{m} T^{2}<F\left(T^{2}\right)$, where

$$
F(t)=\int_{0}^{t} f(s) \mathrm{d} s, \quad \hat{m}=\max _{i \in\{1, \ldots, k\}} m_{i}
$$

Now by assumption (V1), we can fix $\widetilde{m}>0$ such that

$$
\widetilde{m}<\min \left\{m, \liminf _{|x| \rightarrow \infty} V(x)\right\}
$$

and define $\tilde{V}_{\varepsilon}(x)=\max \left\{\widetilde{m}, V_{\varepsilon}(x)\right\}$. Let $H_{\varepsilon}$ be the Hilbert space defined by the completion of $C_{0}^{\infty}\left(\mathbb{R}^{N}, \mathbb{C}\right)$ under the scalar product

$$
\langle u, v\rangle_{\varepsilon}=\operatorname{Re} \int_{\mathbb{R}^{N}}\left(\frac{1}{i} \nabla u-A_{\varepsilon}(x) u\right)\left(\overline{\frac{1}{i} \nabla v-A_{\varepsilon}(x) v}\right)+\tilde{V}_{\varepsilon}(x) u \bar{v} \mathrm{~d} x
$$

and $\|\cdot\|_{\varepsilon}$ the associated norm.

In the present work, we shall prove the following main theorem.

Theorem 1.1. Let $N \geq 3$. Suppose that (A), (V1)-(V2) and (f0)-(f3) hold. Then for any $\varepsilon>0$ sufficiently small, there exists a solution $u_{\varepsilon} \in H_{\varepsilon}$ of $(1.3)$ such that $\left|u_{\varepsilon}\right|$ has $k$ local maximum points $x_{\varepsilon}^{i} \in O^{i}$ satisfying

$$
\lim _{\varepsilon \rightarrow 0} \max _{i=1, \ldots, k} \operatorname{dist}\left(\varepsilon x_{\varepsilon}^{i}, \mathcal{M}^{i}\right)=0
$$

and for which

$$
\left|u_{\varepsilon}(x)\right| \leq C_{1} \exp \left(-C_{2} \min _{i=1, \ldots, k}\left|x-x_{\varepsilon}^{i}\right|\right)
$$

for some positive constants $C_{1}, C_{2}$. Moreover for any sequence $\left(\varepsilon_{n}\right) \subset(0, \varepsilon]$ with $\varepsilon_{n} \rightarrow 0$ there exists a subsequence, still denoted $\left(\varepsilon_{n}\right)$, such that for each $i \in\{1, \ldots, k\}$ there exist $x^{i} \in \mathcal{M}^{i}$ with $\varepsilon_{n} x_{\varepsilon_{n}}^{i} \rightarrow x^{i}$, a constant $w_{i} \in \mathbb{R}$ and $U_{i} \in H^{1}\left(\mathbb{R}^{N}, \mathbb{R}\right)$ a positive least energy solution of

$$
-\Delta U_{i}+m_{i} U_{i}-f\left(\left|U_{i}\right|^{2}\right) U_{i}=0, \quad U_{i} \in H^{1}\left(\mathbb{R}^{N}, \mathbb{R}\right)
$$

for which one has

$$
u_{\varepsilon_{n}}(x)=\sum_{i=1}^{k} U_{i}\left(x-x_{\varepsilon_{n}}^{i}\right) \mathrm{e}^{i\left(w_{i}+A\left(x^{i}\right)\left(x-x_{\varepsilon_{n}}^{i}\right)\right)}+K_{n}(x)
$$

where $K_{n} \in H_{\varepsilon_{n}}$ satisfies $\left\|K_{n}\right\|_{H_{\varepsilon_{n}}}=o(1)$ as $\varepsilon_{n} \rightarrow 0$.

Remark 1.2. Arguing as in [21], we can develop a bootstrap argument, and prove that the solution $u_{\varepsilon} \in H_{\varepsilon}$, found in Theorem 1.1, belongs to $C^{1}\left(\mathbb{R}^{N}, \mathbb{C}\right)$. Indeed, set $u_{\varepsilon}=v+i w$, with $v$, $w$ real valued, we have

$$
-\Delta v+V_{\varepsilon} v=G:=f\left(\left|u_{\varepsilon}\right|^{2}\right) v-2 A_{\varepsilon} \cdot \nabla w-\left|A_{\varepsilon}\right|^{2} v+\left(\operatorname{div} A_{\varepsilon}\right) w
$$

and

$$
-\Delta w+V_{\varepsilon} w=H:=f\left(\left|u_{\varepsilon}\right|^{2}\right) w+2 A_{\varepsilon} \cdot \nabla v-\left|A_{\varepsilon}\right|^{2} w+\left(\operatorname{div} A_{\varepsilon}\right) v .
$$


Since $u_{\varepsilon} \in H_{\varepsilon}$, it follows that for each $K$ bounded set in $\mathbb{R}^{N}, u_{\varepsilon} \in H^{1}(K, \mathbb{C})$. Therefore $v, w \in H^{1}(K, \mathbb{R}) \subset$ $L^{2^{*}}(K, \mathbb{R})$ and by (f2), $G, H \in L^{s}(K, \mathbb{R})$, where $s=\min \left\{2^{*} /(p-1), 2\right\}$. Standard regularity theory implies that $v, w \in W^{2, s}(K)$. If $2 s<N$ we can argue as before and derive that $v, w \in L^{N s /(N-2 s)}(K, \mathbb{R})$ and $\nabla v, \nabla w \in$ $L^{N s /(N-s)}(K, \mathbb{R})$. After a finite number of steps, we have that $v, w \in W^{2, q}(K)$ for any $q \in[1,+\infty[$ and by the Sobolev embedding theorems, $v, w \in C^{1, \alpha}(K, \mathbb{R})$, with $0<\alpha<1$.

Remark 1.3. If we assume the uniqueness of the positive least energy solutions of (1.6) it is not necessary to pass to subsequences to get the decomposition (1.7) in Theorem 1.1.

The proof of Theorem 1.1 follows the approach which is developed in [9] to obtain multi-peak solutions when $A=0$. Roughly speaking we search directly for a solution of (1.3) which consists essentially of $k$ disjoints parts, each part being close to a least energy solution of (1.6) associated to the corresponding $\mathcal{M}^{i}$. Namely in our approach we take into account the shape and location of the solutions we expect to find. Thus on one hand we benefit from the advantage of the Lyapunov-Schmidt reduction type approach, which is to discover the solution around a small neighborhood of a well chosen first approximation. On the other hand our approach, which is purely variational, does not require any uniqueness nor non-degeneracy conditions.

We remark that differently from [9], we need to overcome many additional difficulties which arise for the presence of the magnetic potential. Indeed it is well known that, in general, there is no relationship between the spaces $H_{\varepsilon}$ and $H^{1}\left(\mathbb{R}^{N}, \mathbb{C}\right.$ ), namely $H_{\varepsilon} \not \subset H^{1}\left(\mathbb{R}^{N}, \mathbb{C}\right)$ nor $H^{1}\left(\mathbb{R}^{N}, \mathbb{C}\right) \not \subset H_{\varepsilon}$ (see [27]). This fact explains, for example, the need to restrict to bounded magnetic potentials $A$ when one uses a perturbative approach (see [19]). Our Lemma 2.1 and Corollary 2.2 give some insights of the relationship between $H_{\varepsilon}$ and $H^{1}\left(\mathbb{R}^{N}, \mathbb{C}\right)$ which proves useful in the proof of Theorem 1.1. We also answer positively a question raised by Kurata [35], regarding the equality between the least energy levels for the solutions of

$$
-\Delta U+b U=f\left(|U|^{2}\right) U
$$

when $U$ are sought in $H^{1}\left(\mathbb{R}^{N}, \mathbb{C}\right)$ and $H^{1}\left(\mathbb{R}^{N}, \mathbb{R}\right)$ respectively. See Lemma 2.3 for the precise statement.

In contrast to [5] we do not treat here the cases $N=1$ and $N=2$. For such dimensions applying the approach of [9] is more complex. It can be done when $A=0$ and for the case of a single peak (see [12]) but it is an open question if Theorem 1.1 still holds when $N=1,2$.

The work is organized as follows. In Section 2 we indicate the variational setting and prove some preliminary results. The proof of Theorem 1.1 is derived in Section 3.

\section{VARIATIONAL SETTING AND PRELIMINARY RESUltS}

For any set $B \subset \mathbb{R}^{N}$ and $\varepsilon>0$, let $B_{\varepsilon}=\left\{x \in \mathbb{R}^{N} \mid \varepsilon x \in B\right\}$.

Lemma 2.1. Let $K \subset \mathbb{R}^{N}$ be an arbitrary fixed bounded domain. Assume that $A$ is bounded on $K$ and $0<\alpha \leq V \leq \beta$ on $K$ for some $\alpha, \beta>0$. Then, for any fixed $\varepsilon \in[0,1]$, the norm

$$
\|u\|_{K_{\varepsilon}}^{2}=\int_{K_{\varepsilon}}\left|\left(\frac{1}{i} \nabla-A_{\varepsilon}(y)\right) u\right|^{2}+V_{\varepsilon}(y)|u|^{2} \mathrm{~d} y
$$

is equivalent to the usual norm on $H^{1}\left(K_{\varepsilon}, \mathbb{C}\right)$. Moreover these equivalences are uniform, i.e. there exist $c_{1}, c_{2}>0$ independent of $\varepsilon \in[0,1]$ such that

$$
c_{1}\|u\|_{K_{\varepsilon}} \leq\|u\|_{H^{1}\left(K_{\varepsilon}, \mathbb{C}\right)} \leq c_{2}\|u\|_{K_{\varepsilon}} .
$$

Proof. Our proof is inspired by the one of Lemma 2.3 in [3]. We have

$$
\int_{K_{\varepsilon}}\left|A_{\varepsilon}(y) u\right|^{2} \mathrm{~d} y \leq\|A\|_{L^{\infty}(K)} \int_{K_{\varepsilon}}|u|^{2} \mathrm{~d} y
$$


and

$$
\int_{K_{\varepsilon}} V_{\varepsilon}(y)|u|^{2} \mathrm{~d} y \leq\|V\|_{L^{\infty}(K)} \int_{K_{\varepsilon}}|u|^{2} \mathrm{~d} y
$$

Hence

$$
\begin{aligned}
\int_{K_{\varepsilon}}\left|\frac{1}{i} \nabla u-A_{\varepsilon}(y) u\right|^{2}+V_{\varepsilon}(y)|u|^{2} \mathrm{~d} y & \leq \int_{K_{\varepsilon}} 2\left(|\nabla u|^{2}+\left|A_{\varepsilon}(y) u\right|^{2}\right)+V_{\varepsilon}(y)|u|^{2} \mathrm{~d} y \\
& \leq 2 \int_{K_{\varepsilon}}|\nabla u|^{2} \mathrm{~d} y+\left(2\|A\|_{L^{\infty}(K)}+\|V\|_{L^{\infty}(K)}\right) \int_{K_{\varepsilon}}|u|^{2} \mathrm{~d} y .
\end{aligned}
$$

To prove the other inequality note that

$$
\int_{K_{\varepsilon}}\left|\frac{1}{i} \nabla u-A_{\varepsilon}(y) u\right|^{2}+V_{\varepsilon}(y)|u|^{2} \mathrm{~d} y \geq\left.\int_{K_{\varepsilon}}|| \nabla u\right|^{2}-\left|A_{\varepsilon}(y) u\right|^{2}+V_{\varepsilon}(y)|u|^{2} \mathrm{~d} y .
$$

We shall prove that, for some $d>0$ independent of $\varepsilon \in[0,1]$,

$$
\left.\int_{K_{\varepsilon}}|| \nabla u|-| A_{\varepsilon}(y) u\right|^{2}+V_{\varepsilon}(y)|u|^{2} \mathrm{~d} y \geq d \int_{K_{\varepsilon}}|\nabla u|^{2}+|u|^{2} \mathrm{~d} y .
$$

Arguing by contradiction we assume that there exist sequences $\left(\varepsilon_{n}\right) \subset[0,1]$ and $\left(u_{\varepsilon_{n}}\right) \subset H^{1}\left(K_{\varepsilon_{n}}, \mathbb{C}\right)$ with $\left\|u_{\varepsilon_{n}}\right\|_{H^{1}\left(K_{\varepsilon_{n}}, \mathbb{C}\right)}=1$ such that

$$
\int_{K_{\varepsilon_{n}}}|| \nabla u_{\varepsilon_{n}}|-| A_{\varepsilon_{n}}(y) u_{\varepsilon_{n}}||^{2}+V_{\varepsilon_{n}}(y)\left|u_{\varepsilon_{n}}\right|^{2} \mathrm{~d} y<\frac{1}{n}
$$

Clearly $\left(u_{\varepsilon_{n}}\right) \subset H^{1}\left(\mathbb{R}^{N}, \mathbb{C}\right)$ and $\left\|u_{\varepsilon_{n}}\right\|_{H^{1}\left(\mathbb{R}^{N}, \mathbb{C}\right)}=1$. Passing to a subsequence, $u_{\varepsilon_{n}} \rightarrow u$ weakly in $H^{1}\left(\mathbb{R}^{N}, \mathbb{C}\right)$. Since $V_{\varepsilon_{n}} \geq \alpha>0$ on $K_{\varepsilon_{n}}$ we see from (2.2) that necessarily

$$
\int_{K_{\varepsilon_{n}}}\left|u_{\varepsilon_{n}}\right|^{2} \mathrm{~d} y \rightarrow 0
$$

Thus $u_{\varepsilon_{n}} \rightarrow 0$ in $L^{2}\left(\mathbb{R}^{N}, \mathbb{C}\right)$ strongly and in particular $u_{\varepsilon_{n}} \rightarrow 0$ in $H^{1}\left(\mathbb{R}^{N}, \mathbb{C}\right)$. Now

$$
\left.\int_{K_{\varepsilon_{n}}}|| \nabla u_{\varepsilon_{n}}|-| A_{\varepsilon_{n}}(y) u_{\varepsilon_{n}}\right|^{2} \mathrm{~d} y=\int_{K_{\varepsilon_{n}}}\left|\nabla u_{\varepsilon_{n}}\right|^{2}-2\left|A_{\varepsilon_{n}}(y) u_{\varepsilon_{n}}\right|\left|\nabla u_{\varepsilon_{n}}\right|+\left|A_{\varepsilon_{n}}(y) u_{\varepsilon_{n}}\right|^{2} \mathrm{~d} y
$$

with

$$
\int_{K_{\varepsilon_{n}}}\left|A_{\varepsilon_{n}}(y) u_{\varepsilon_{n}}\right|\left|\nabla u_{\varepsilon_{n}}\right| \mathrm{d} y \rightarrow 0
$$

Indeed we have

$$
\begin{aligned}
\int_{K_{\varepsilon_{n}}}\left|A_{\varepsilon_{n}}(y) u_{\varepsilon_{n}}\right|\left|\nabla u_{\varepsilon_{n}}\right| \mathrm{d} y & \leq\left(\int_{K_{\varepsilon_{n}}}\left|A_{\varepsilon_{n}}(y) u_{\varepsilon_{n}}\right|^{2} \mathrm{~d} y\right)^{\frac{1}{2}}\left(\int_{K_{\varepsilon_{n}}}\left|\nabla u_{\varepsilon_{n}}\right|^{2} \mathrm{~d} y\right)^{\frac{1}{2}} \\
& \leq\|A\|_{L^{\infty}(K)}\left(\int_{K_{\varepsilon_{n}}}\left|u_{\varepsilon_{n}}\right|^{2} \mathrm{~d} y\right)^{\frac{1}{2}} .
\end{aligned}
$$


Thus

$$
0=\left.\limsup _{n \rightarrow+\infty} \int_{K_{\varepsilon_{n}}}|| \nabla u_{\varepsilon_{n}}|-| A_{\varepsilon_{n}}(y) u_{\varepsilon_{n}}\right|^{2} \mathrm{~d} y \geq \limsup _{n \rightarrow+\infty} \int_{K_{\varepsilon_{n}}}\left|\nabla u_{\varepsilon_{n}}\right|^{2} \mathrm{~d} y
$$

But this is impossible since otherwise we would have $u_{\varepsilon_{n}} \rightarrow 0$ strongly in $H^{1}\left(\mathbb{R}^{N}, \mathbb{C}\right)$.

From Lemma 2.1 we immediately deduce the following corollary.

Corollary 2.2. Retain the setting of Lemma 2.1.

(i) If $K$ is compact, for any $\varepsilon \in(0,1]$ the norm

$$
\|u\|_{K}^{2}:=\int_{K}\left|\left(\frac{1}{i} \nabla-A_{\varepsilon}(y)\right) u\right|^{2}+V_{\varepsilon}(y)|u|^{2} \mathrm{~d} y
$$

is uniformly equivalent to the usual norm on $H^{1}(K, \mathbb{C})$.

(ii) For $A_{0} \in \mathbb{R}^{N}$ and $b>0$ fixed, the norm

$$
\|u\|^{2}:=\int_{\mathbb{R}^{N}}\left|\left(\frac{1}{i} \nabla-A_{0}\right) u\right|^{2}+b|u|^{2} \mathrm{~d} y
$$

is equivalent to the usual norm on $H^{1}\left(\mathbb{R}^{N}, \mathbb{C}\right)$.

(iii) If $\left(u_{\varepsilon_{n}}\right) \subset H^{1}\left(\mathbb{R}^{N}, \mathbb{C}\right)$ satisfies $u_{\varepsilon_{n}}=0$ on $\mathbb{R}^{N} \backslash K_{\varepsilon_{n}}$ for any $n \in \mathbb{N}$ and $u_{\varepsilon_{n}} \rightarrow u$ in $H^{1}\left(\mathbb{R}^{N}, \mathbb{C}\right)$ then $\left\|u_{\varepsilon_{n}}-u\right\|_{\varepsilon_{n}} \rightarrow 0$ as $n \rightarrow \infty$.

Proof. Indeed (i) is trivial, to see (ii) just put $\varepsilon=0$ in Lemma 2.1. Now (iii) follows from the uniformity of the equivalence derived in Lemma 2.1.

For future reference we recall the following

Diamagnetic inequality: For every $u \in H_{\varepsilon}$,

$$
\left|\left(\frac{\nabla}{i}-A_{\varepsilon}\right) u\right| \geq|\nabla| u||, \quad \text { a.e. in } \mathbb{R}^{N}
$$

See [27] for a proof. As a consequence of $(2.3),|u| \in H^{1}\left(\mathbb{R}^{N}, \mathbb{R}\right)$ for any $u \in H_{\varepsilon}$.

Now we define

$$
\mathcal{M}=\bigcup_{i=1}^{k} \mathcal{M}^{i}, \quad O=\bigcup_{i=1}^{k} O^{i}
$$

and for any set $B \subset \mathbb{R}^{N}$ and $\alpha>0, B^{\delta}=\left\{x \in \mathbb{R}^{N} \mid \operatorname{dist}(x, B) \leq \delta\right\}$. For $u \in H_{\varepsilon}$, let

$$
\mathcal{F}_{\varepsilon}(u)=\frac{1}{2} \int_{\mathbb{R}^{N}}\left|D^{\varepsilon} u\right|^{2}+V_{\varepsilon}|u|^{2} \mathrm{~d} y-\int_{\mathbb{R}^{N}} F\left(|u|^{2}\right) \mathrm{d} y
$$

where we set $D^{\varepsilon}=\left(\frac{\nabla}{i}-A_{\varepsilon}\right)$. Define

$$
\chi_{\varepsilon}(y)=\left\{\begin{array}{ll}
0 & \text { if } y \in O_{\varepsilon}, \\
\varepsilon^{-6 / \mu} & \text { if } y \notin O_{\varepsilon},
\end{array} \quad \chi_{\varepsilon}^{i}(y)= \begin{cases}0 & \text { if } y \in\left(O^{i}\right)_{\varepsilon}, \\
\varepsilon^{-6 / \mu} & \text { if } y \notin\left(O^{i}\right)_{\varepsilon},\end{cases}\right.
$$

and

$$
Q_{\varepsilon}(u)=\left(\int_{\mathbb{R}^{N}} \chi_{\varepsilon}|u|^{2} \mathrm{~d} y-1\right)_{+}^{\frac{p+2}{2}}, \quad Q_{\varepsilon}^{i}(u)=\left(\int_{\mathbb{R}^{N}} \chi_{\varepsilon}^{i}|u|^{2} \mathrm{~d} y-1\right)_{+}^{\frac{p+2}{2}} .
$$


The functional $Q_{\varepsilon}$ will act as a penalization to force the concentration phenomena to occur inside $O$. This type of penalization was first introduced in [11]. Finally we define the functionals $\Gamma_{\varepsilon}, \Gamma_{\varepsilon}^{1}, \ldots, \Gamma_{\varepsilon}^{k}: H_{\varepsilon} \rightarrow \mathbb{R}$ by

$$
\Gamma_{\varepsilon}(u)=\mathcal{F}_{\varepsilon}(u)+Q_{\varepsilon}(u), \quad \Gamma_{\varepsilon}^{i}(u)=\mathcal{F}_{\varepsilon}(u)+Q_{\varepsilon}^{i}(u), i=1, \ldots, k
$$

It is easy to check, under our assumptions, and using the diamagnetic inequality (2.3), that the functionals $\Gamma_{\varepsilon}, \Gamma_{\varepsilon}^{i} \in C^{1}\left(H_{\varepsilon}\right)$. So a critical point of $\mathcal{F}_{\varepsilon}$ corresponds to a solution of (1.3). To find solutions of (1.3) which concentrate in $O$ as $\varepsilon \rightarrow 0$, we shall look for a critical point of $\Gamma_{\varepsilon}$ for which $Q_{\varepsilon}$ is zero.

Let us consider for $a>0$ the scalar limiting equation of (1.3)

$$
-\Delta u+a u=f\left(|u|^{2}\right) u, \quad u \in H^{1}\left(\mathbb{R}^{N}, \mathbb{R}\right) .
$$

Solutions of (2.7) correspond to critical points of the limiting functional $L_{a}: H^{1}\left(\mathbb{R}^{N}, \mathbb{R}\right) \rightarrow \mathbb{R}$ defined by

$$
L_{a}(u)=\frac{1}{2} \int_{\mathbb{R}^{N}}\left(|\nabla u|^{2}+a|u|^{2}\right) \mathrm{d} y-\int_{\mathbb{R}^{N}} F\left(|u|^{2}\right) \mathrm{d} y .
$$

In [6], Berestycki and Lions proved that, for any $a>0$, under the assumptions (f0)-(f2) and (f3) with $\hat{m}=a$, there exists a least energy solution and that each solution $U$ of (2.7) satisfies the Pohozaev's identity

$$
\frac{N-2}{2} \int_{\mathbb{R}^{N}}|\nabla U|^{2} \mathrm{~d} y+N \int_{\mathbb{R}^{N}} a \frac{|U|^{2}}{2}-F\left(|U|^{2}\right) \mathrm{d} y=0 .
$$

From this we immediately deduce that, for any solution $U$ of $(2.7)$,

$$
\frac{1}{N} \int_{\mathbb{R}^{N}}|\nabla U|^{2} \mathrm{~d} y=L_{a}(U)
$$

We also consider the complex valued equation, for $a>0$,

$$
-\Delta u+a u=f\left(|u|^{2}\right) u, \quad u \in H^{1}\left(\mathbb{R}^{N}, \mathbb{C}\right) .
$$

In turn solutions of (2.11) correspond to critical points of the functional $L_{a}^{c}: H^{1}\left(\mathbb{R}^{N}, \mathbb{C}\right) \rightarrow \mathbb{R}$, defined by

$$
L_{a}^{c}(v)=\frac{1}{2} \int_{\mathbb{R}^{N}}\left(|\nabla v|^{2}+a|v|^{2}\right) \mathrm{d} y-\int_{\mathbb{R}^{N}} F\left(|v|^{2}\right) \mathrm{d} y .
$$

In [43] the Pohozaev's identity (2.9) and thus (2.10) is given for complex-valued solutions of (2.11). The following result relates the least energy levels of (2.7) and (2.11) and positively answers to a question of Kurata [35] (see also [42] for some elements of proof in that direction). When $N=2$ we say that (f2) holds if

$$
\text { for all } \alpha>0 \text { there exists } C_{\alpha}>0 \text { such that }\left|f\left(t^{2}\right)\right| \leq C_{\alpha} \mathrm{e}^{\alpha t^{2}} \text {, for all } t \geq 0 \text {. }
$$

Lemma 2.3. Suppose that (f0)-(f2) and (f3) with $\hat{m}=a$ hold and that $N \geq 2$. Let $E_{a}$ and $E_{a}^{c}$ denote the least energy levels corresponding to equations (2.7) and (2.11). Then

$$
E_{a}=E_{a}^{c}
$$

Moreover any least energy solution of (2.11) has the form $\mathrm{e}^{i \tau} U$ where $U$ is a positive least energy solution of (2.7) and $\tau \in \mathbb{R}$. 
Proof. The inequality $E_{a}^{c} \leq E_{a}$ is obvious and thus to establish that $E_{a}^{c}=E_{a}$ we just need to prove that $E_{a} \leq E_{a}^{c}$.

We know from [43] that each solution of (2.11) satisfies the Pohozaev's identity $P(u)=0$ where $P: H^{1}\left(\mathbb{R}^{N}, \mathbb{C}\right) \rightarrow$ $\mathbb{R}$ is defined by

$$
P(u)=\frac{N-2}{2} \int_{\mathbb{R}^{N}}|\nabla u|^{2} \mathrm{~d} y+N \int_{\mathbb{R}^{N}} a \frac{|u|^{2}}{2}-F\left(|u|^{2}\right) \mathrm{d} y .
$$

By Lemma 3.1 of [32] we have that

$$
\inf _{\substack{u \in H^{1}\left(\mathbb{R}^{N}, \mathbb{R}\right) \\ P(u)=0}} L_{a}(u)=E_{a} .
$$

Also it is well known (see for example [31]) that for any $u \in H^{1}(\mathbb{R}, \mathbb{C}$ ) one has

$$
\int_{\mathbb{R}^{N}}|\nabla| u||^{2} \mathrm{~d} y \leq \int_{\mathbb{R}^{N}}|\nabla u|^{2} \mathrm{~d} y
$$

Now let $U$ be a solution of (2.11). If $N=2$ we see from the definition of $P$ that $P(|U|)=0$ and from (2.15) that $L_{a}(|U|) \leq L_{a}^{c}(U)$. Thus $E_{a} \leq E_{a}^{c}$ follows from (2.14). In addition, if $U$ is a least energy solution of (2.11), necessarily

$$
\left.\int_{\mathbb{R}^{N}}|\nabla| U\right|^{2} \mathrm{~d} y=\int_{\mathbb{R}^{N}}|\nabla U|^{2} \mathrm{~d} y
$$

and $|U|$ is a least energy solution of (2.7). If $N \geq 3$ we see from (2.15) that either

(i) $P(|U|)=0$ and $L_{a}(|U|)=L_{a}^{c}(U)$.

(ii) $P(|U|)<0$ and there exists $\theta \in] 0,1\left[\right.$ such that, for $U_{\theta}(\cdot)=U(\cdot / \theta)$ we have $P\left(\left|U_{\theta}\right|\right)=0$. Then, since $P\left(\left|U_{\theta}\right|\right)=0$, it follows that

$$
L_{a}\left(\left|U_{\theta}\right|\right)=\frac{1}{N} \int_{\mathbb{R}^{N}}|\nabla| U_{\theta}||^{2} \mathrm{~d} y=\frac{\theta^{N-2}}{N} \int_{\mathbb{R}^{N}}|\nabla| U||^{2} \mathrm{~d} y
$$

and thus

$$
L_{a}\left(\left|U_{\theta}\right|\right)<\frac{1}{N} \int_{\mathbb{R}^{N}}|\nabla| U||^{2} \mathrm{~d} y \leq \frac{1}{N} \int_{\mathbb{R}^{N}}|\nabla U|^{2} \mathrm{~d} y=L_{a}^{c}(U) .
$$

In both cases we deduce from (2.14) that $E_{a} \leq E_{a}^{c}$. In addition if $U$ is a least energy solution of (2.11) then (2.16) holds and in particular $|U|$ is a least energy solution of (2.7).

Now, for any $N \geq 2$, let $U$ be a least energy solution of (2.11). Since $|U|$ is a solution of (2.7) we get by elliptic regularity theory and the maximum principle that $|U| \in C^{1}\left(\mathbb{R}^{N}, \mathbb{R}\right)$ and $|U|>0$. At this point, using (2.16), the rest of the proof of the lemma is exactly the same as the proof of Theorem 4.1 in [31].

Remark 2.4. When $N=1$ conditions which assure that (2.7) has, up to translation, a unique positive solution are given in [6] (see also [33] for alternative conditions). Now following the proof of Theorem 8.1.6 in [14] we deduce that any solution of (2.11) is of the form $\mathrm{e}^{i \theta} \rho$ where $\theta \in \mathbb{R}$ and $\rho>0$ is a solution of (2.7). Thus, under the assumptions of [6,33], the result of Lemma 2.3 also holds when $N=1$ and the positive least energy solution is unique.

Now let $S_{a}$ be the set of least energy solutions $U$ of (2.11) satisfying

$$
|U(0)|=\max _{y \in \mathbb{R}^{N}}|U(y)| .
$$

By standard regularity any solution of $(2.11)$ is at least $C^{1}$. Since $f$ is not assumed to be locally Hölder continuous we do not know, in contrast to [5], if any least energy solution is radially symmetric. However the following compactness result can still be proved. 
Proposition 2.5. For each $a>0$ and $N \geq 3, S_{a}$ is compact in $H^{1}\left(\mathbb{R}^{N}, \mathbb{C}\right)$. Moreover, there exist $C, c>0$, independent of $U \in S_{a}$, such that

$$
|U(y)| \leq C \exp (-c|y|)
$$

Proof. In [7], the same results are proved when $S_{a}$ is restricted to real solutions. Since, by Lemma 2.3, any least energy solution of (2.11) is of the form $\mathrm{e}^{i \tau} \tilde{U}$ with $\tilde{U}$ a least energy solution of (2.7) it proves the lemma.

\section{Proof of Theorem 1.1}

Let

$$
\delta=\frac{1}{10} \min \left\{\operatorname{dist}\left(\mathcal{M}, \mathbb{R}^{N} \backslash O\right), \min _{i \neq j} \operatorname{dist}\left(O_{i}, O_{j}\right), \operatorname{dist}(O, Z)\right\} .
$$

We fix a $\beta \in(0, \delta)$ and a cutoff $\varphi \in C_{0}^{\infty}\left(\mathbb{R}^{N}\right)$ such that $0 \leq \varphi \leq 1, \varphi(y)=1$ for $|y| \leq \beta$ and $\varphi(y)=0$ for $|y| \geq 2 \beta$. Also, setting $\varphi_{\varepsilon}(y)=\varphi(\varepsilon y)$ for each $x_{i} \in\left(\mathcal{M}^{i}\right)^{\beta}$ and $U_{i} \in S_{m_{i}}$, we define

$$
U_{\varepsilon}^{x_{1}, \ldots, x_{k}}(y)=\sum_{i=1}^{k} \mathrm{e}^{i A\left(x_{i}\right)\left(y-\frac{x_{i}}{\varepsilon}\right)} \varphi_{\varepsilon}\left(y-\frac{x_{i}}{\varepsilon}\right) U_{i}\left(y-\frac{x_{i}}{\varepsilon}\right) .
$$

We will find a solution, for sufficiently small $\varepsilon>0$, near the set

$$
X_{\varepsilon}=\left\{U_{\varepsilon}^{x_{1}, \ldots, x_{k}}(y) \mid x_{i} \in\left(\mathcal{M}^{i}\right)^{\beta} \text { and } U_{i} \in S_{m_{i}} \text { for each } i=1, \ldots, k\right\} .
$$

For each $i \in\{1, \ldots, k\}$ we fix an arbitrary $x_{i} \in \mathcal{M}^{i}$ and an arbitrary $U_{i} \in S_{m_{i}}$ and we define

$$
W_{\varepsilon}^{i}(y)=\mathrm{e}^{i A\left(x_{i}\right)\left(y-\frac{x_{i}}{\varepsilon}\right)} \varphi_{\varepsilon}\left(y-\frac{x_{i}}{\varepsilon}\right) U_{i}\left(y-\frac{x_{i}}{\varepsilon}\right) .
$$

Setting

$$
W_{\varepsilon, t}^{i}(y)=\mathrm{e}^{i A\left(x_{i}\right)\left(y-\frac{x_{i}}{\varepsilon}\right)} \varphi_{\varepsilon}\left(y-\frac{x_{i}}{\varepsilon}\right) U_{i}\left(\frac{y}{t}-\frac{x_{i}}{\varepsilon t}\right),
$$

we see that $\lim _{t \rightarrow 0}\left\|W_{\varepsilon, t}^{i}\right\|_{\varepsilon}=0$ (recall that $N \geq 3$ ) and that $\Gamma_{\varepsilon}\left(W_{\varepsilon, t}^{i}\right)=\mathcal{F}_{\varepsilon}\left(W_{\varepsilon, t}^{i}\right)$ for $t \geq 0$. In the next proposition we shall prove that there exists $T_{i}>0$ such that $\Gamma_{\varepsilon}\left(W_{\varepsilon, T_{i}}^{i}\right)<-2$ for any $\varepsilon>0$ sufficiently small. Assuming this holds true, let $\gamma_{\varepsilon}^{i}(s)=W_{\varepsilon, s}^{i}$ for $s>0$ and $\gamma_{\varepsilon}^{i}(0)=0$. For $s=\left(s_{1}, \ldots, s_{k}\right) \in T=\left[0, T_{1}\right] \times \ldots \times\left[0, T_{k}\right]$ we define

$$
\gamma_{\varepsilon}(s)=\sum_{i=1}^{k} W_{\varepsilon, s_{i}}^{i} \quad \text { and } \quad D_{\varepsilon}=\max _{s \in T} \Gamma_{\varepsilon}\left(\gamma_{\varepsilon}(s)\right)
$$

Finally for each $i \in\{1, \ldots, k\}$, let $E_{m_{i}}=L_{m_{i}}^{c}(U)$ for $U \in S_{m_{i}}$. In what follows, we set $E_{m}=\min _{i \in\{1, \ldots, k\}} E_{m_{i}}$ and $E=\sum_{i=1}^{k} E_{m_{i}}$. For a set $A \subset H_{\varepsilon}$ and $\alpha>0$, we let $A^{\alpha}=\left\{u \in H_{\varepsilon} \mid\|u-A\|_{\varepsilon} \leq \alpha\right\}$.

Proposition 3.1. We have

(i) $\lim _{\varepsilon \rightarrow 0} D_{\varepsilon}=E$;

(ii) $\limsup _{\varepsilon \rightarrow 0} \max _{s \in \partial T} \Gamma_{\varepsilon}\left(\gamma_{\varepsilon}(s)\right) \leq \tilde{E}=\max \left\{E-E_{m_{i}} \mid i=1, \ldots, k\right\}<E$;

(iii) for each $d>0$, there exists $\alpha>0$ such that for sufficiently small $\varepsilon>0$,

$$
\Gamma_{\varepsilon}\left(\gamma_{\varepsilon}(s)\right) \geq D_{\varepsilon}-\alpha \text { implies that } \gamma_{\varepsilon}(s) \in X_{\varepsilon}^{d / 2} .
$$


662

S. CINGOLANI ET AL.

Proof. Since $\operatorname{supp}\left(\gamma_{\varepsilon}(s)\right) \subset \mathcal{M}_{\varepsilon}^{2 \beta}$ for each $s \in T$, it follows that $\Gamma_{\varepsilon}\left(\gamma_{\varepsilon}(s)\right)=\mathcal{F}_{\varepsilon}\left(\gamma_{\varepsilon}(s)\right)=\sum_{i=1}^{k} \mathcal{F}_{\varepsilon}\left(\gamma_{\varepsilon}^{i}(s)\right)$. Now, for each $i \in\{1, \ldots, k\}$, we claim that

$$
\lim _{\varepsilon \rightarrow 0} \int_{\mathbb{R}^{N}}\left|\left(\frac{\nabla}{i}-A_{\varepsilon}(y)\right) W_{\varepsilon, s_{i}}^{i}\right|^{2} \mathrm{~d} y=s_{i}^{N-2} \int_{\mathbb{R}^{N}}\left|\nabla U_{i}\right|^{2} \mathrm{~d} y
$$

Indeed

$$
\int_{\mathbb{R}^{N}}\left|\left(\frac{\nabla}{i}-A_{\varepsilon}(y)\right) W_{\varepsilon, s_{i}}^{i}\right|^{2} \mathrm{~d} y=\int_{\mathbb{R}^{N}}\left(\left|\nabla W_{\varepsilon, s_{i}}^{i}\right|^{2}+\left|A_{\varepsilon}(y)\right|^{2}\left|W_{\varepsilon, s_{i}}^{i}\right|^{2}-2 \operatorname{Re}\left[\frac{1}{i} \nabla W_{\varepsilon, s_{i}}^{i} \cdot A_{\varepsilon}(y) \overline{W_{\varepsilon, s_{i}}^{i}}\right]\right) \mathrm{d} y
$$

with

$$
\begin{aligned}
\int_{\mathbb{R}^{N}}\left|\nabla W_{\varepsilon, s_{i}}^{i}\right|^{2} \mathrm{~d} y= & \int_{\mathbb{R}^{N}} \mid i A\left(x_{i}\right) U_{i}\left(\frac{y}{s_{i}}-\frac{x_{i}}{\varepsilon s_{i}}\right) \varphi_{\varepsilon}\left(y-\frac{x_{i}}{\varepsilon}\right)+\frac{1}{s_{i}} \nabla U_{i}\left(\frac{y}{s_{i}}-\frac{x_{i}}{\varepsilon s_{i}}\right) \varphi_{\varepsilon}\left(y-\frac{x_{i}}{\varepsilon}\right) \\
& +\left.\varepsilon \nabla_{\tau} \varphi\left(\varepsilon y-x_{i}\right) U_{i}\left(\frac{y}{s_{i}}-\frac{x_{i}}{\varepsilon s_{i}}\right)\right|^{2} \mathrm{~d} y \\
= & \int_{\mathbb{R}^{N}}\left|A\left(x_{i}\right)\right|^{2}\left|U_{i}\left(\frac{y}{s_{i}}\right)\right|^{2}\left|\varphi_{\varepsilon}(y)\right|^{2} \mathrm{~d} y \\
& +\int_{\mathbb{R}^{N}}\left|\frac{1}{s_{i}} \nabla U_{i}\left(\frac{y}{s_{i}}\right) \varphi_{\varepsilon}(y)+\varepsilon \nabla_{\tau} \varphi(\varepsilon y) U_{i}\left(\frac{y}{s_{i}}\right)\right|^{2} \mathrm{~d} y .
\end{aligned}
$$

Moreover we have

$$
\int_{\mathbb{R}^{N}}\left|A_{\varepsilon}(y)\right|^{2}\left|W_{\varepsilon, s_{i}}^{i}\right|^{2} \mathrm{~d} y=\int_{\mathbb{R}^{N}}\left|A_{\varepsilon}(y)\right|^{2}\left|U_{i}\left(\frac{y}{s_{i}}-\frac{x_{i}}{\varepsilon s_{i}}\right)\right|^{2}\left|\varphi_{\varepsilon}\left(y-\frac{x_{i}}{\varepsilon}\right)\right|^{2} \mathrm{~d} y
$$

and

$$
\int_{\mathbb{R}^{N}} \operatorname{Re}\left[\frac{1}{i} \nabla W_{\varepsilon, s_{i}}^{i} \cdot A_{\varepsilon}(y) \overline{W_{\varepsilon, s_{i}}^{i}}\right] \mathrm{d} y=\int_{\mathbb{R}^{N}} A_{\varepsilon}\left(x_{i}\right) \cdot A_{\varepsilon}(y)\left|U_{i}\left(\frac{y}{s_{i}}-\frac{x_{i}}{\varepsilon s_{i}}\right)\right|^{2}\left|\varphi_{\varepsilon}\left(y-\frac{x_{i}}{\varepsilon}\right)\right|^{2} \mathrm{~d} y .
$$

Since, as $\varepsilon \rightarrow 0$,

$$
\int_{\mathbb{R}^{N}}\left|A_{\varepsilon}(y)\right|^{2}\left|U_{i}\left(\frac{y}{s_{i}}-\frac{x_{i}}{\varepsilon s_{i}}\right)\right|^{2}\left|\varphi_{\varepsilon}\left(y-\frac{x_{i}}{\varepsilon}\right)\right|^{2} \mathrm{~d} y \rightarrow \int_{\mathbb{R}^{N}}\left|A\left(x_{i}\right)\right|^{2}\left|U_{i}\left(\frac{y}{s_{i}}\right)\right|^{2} \mathrm{~d} y,
$$

and

$$
\int_{\mathbb{R}^{N}} A_{\varepsilon}\left(x_{i}\right) \cdot A_{\varepsilon}(y)\left|U_{i}\left(\frac{y}{s_{i}}-\frac{x_{i}}{\varepsilon s_{i}}\right)\right|^{2}\left|\varphi_{\varepsilon}\left(y-\frac{x_{i}}{\varepsilon}\right)\right|^{2} \mathrm{~d} y \rightarrow \int_{\mathbb{R}^{N}}\left|A\left(x_{i}\right)\right|^{2}\left|U_{i}\left(\frac{y}{s_{i}}\right)\right|^{2} \mathrm{~d} y,
$$

taking into account (3.2)-(3.5) it follows that,

$$
\int_{\mathbb{R}^{N}}\left|\left(\frac{\nabla}{i}-A_{\varepsilon}(y)\right) W_{\varepsilon, s_{i}}^{i}\right|^{2} \mathrm{~d} y \rightarrow \frac{1}{s_{i}^{2}} \int_{\mathbb{R}^{N}}\left|\nabla U_{i}\left(\frac{y}{s_{i}}\right)\right|^{2} \mathrm{~d} y=s_{i}^{N-2} \int_{\mathbb{R}^{N}}\left|\nabla U_{i}\right|^{2} \mathrm{~d} y
$$


and this proves (3.1). Similarly using the exponential decay of $U_{i}$ we have, as $\varepsilon \rightarrow 0$,

$$
\begin{gathered}
\int_{\mathbb{R}^{N}} V_{\varepsilon}(y)\left|W_{\varepsilon, s_{i}}^{i}\right|^{2} \mathrm{~d} y \rightarrow \int_{\mathbb{R}^{N}} m_{i}\left|U_{i}\left(\frac{y}{s_{i}}\right)\right|^{2} \mathrm{~d} y=m_{i} s_{i}^{N} \int_{\mathbb{R}^{N}}\left|U_{i}\right|^{2} \mathrm{~d} y \\
\int_{\mathbb{R}^{N}} F\left(\left|W_{\varepsilon, s_{i}}^{i}\right|^{2}\right) \mathrm{d} y \rightarrow \int_{\mathbb{R}^{N}} F\left(\left|U_{i}\left(\frac{y}{s_{i}}\right)\right|^{2}\right) \mathrm{d} y=s_{i}^{N} \int_{\mathbb{R}^{N}} F\left(\left|U_{i}\right|^{2}\right) \mathrm{d} y .
\end{gathered}
$$

Thus, from (3.1), (3.7) and (3.8),

$$
\begin{aligned}
\mathcal{F}_{\varepsilon}\left(\gamma_{\varepsilon}^{i}\left(s_{i}\right)\right) & =\frac{1}{2} \int_{\mathbb{R}^{N}}\left|\left(\frac{\nabla}{i}-A_{\varepsilon}(y)\right) \gamma_{\varepsilon}^{i}\left(s_{i}\right)\right|^{2} \mathrm{~d} y+V_{\varepsilon}(y)\left|\gamma_{\varepsilon}^{i}\left(s_{i}\right)\right|^{2} \mathrm{~d} y-\int_{\mathbb{R}^{N}} F\left(\left|\gamma_{\varepsilon}^{i}\left(s_{i}\right)\right|^{2}\right) \mathrm{d} y \\
& =\frac{s_{i}^{N-2}}{2} \int_{\mathbb{R}^{N}}\left|\nabla U_{i}\right|^{2} \mathrm{~d} y+s_{i}^{N} \int_{\mathbb{R}^{N}} \frac{1}{2} m_{i}\left|U_{i}\right|^{2}-F\left(\left|U_{i}\right|^{2}\right) \mathrm{d} y+o(1) .
\end{aligned}
$$

Then, from the Pohozaev identity (2.9), we see that

$$
\mathcal{F}_{\varepsilon}\left(\gamma_{\varepsilon}^{i}\left(s_{i}\right)\right)=\left(\frac{s_{i}^{N-2}}{2}-\frac{N-2}{2 N} s_{i}^{N}\right) \int_{\mathbb{R}^{N}}\left|\nabla U_{i}\right|^{2} \mathrm{~d} y+o(1) .
$$

Also

$$
\max _{t \in(0, \infty)}\left(\frac{t^{N-2}}{2}-\frac{N-2}{2 N} t^{N}\right) \int_{\mathbb{R}^{N}}\left|\nabla U_{i}\right|^{2} \mathrm{~d} y=E_{m_{i}}
$$

At this point we deduce that (i) and (ii) hold. Clearly also the existence of a $T_{i}>0$ such that $\Gamma_{\varepsilon}\left(W_{\varepsilon, T_{i}}^{i}\right)<-2$ is justified. To conclude we just observe that for $g(t)=\frac{t^{N-2}}{2}-\frac{N-2}{2 N} t^{N}$,

$$
g^{\prime}(t) \begin{cases}>0 & \text { for } t \in(0,1) \\ =0 & \text { for } t=1 \\ <0 & \text { for } t>1\end{cases}
$$

and $g^{\prime \prime}(1)=2-N<0$.

Now let

$$
\Phi_{\varepsilon}^{i}=\left\{\gamma \in C\left(\left[0, T_{i}\right], H_{\varepsilon}\right) \mid \gamma\left(s_{i}\right)=\gamma_{\varepsilon}^{i}\left(s_{i}\right) \text { for } s_{i}=0 \text { or } T_{i}\right\}
$$

and

$$
C_{\varepsilon}^{i}=\inf _{\gamma \in \Phi_{\varepsilon}^{i}} \max _{s_{i} \in\left[0, T_{i}\right]} \Gamma_{\varepsilon}^{i}\left(\gamma\left(s_{i}\right)\right)
$$

For future reference we need the following estimate.

Proposition 3.2. For $i=1, \ldots, k$,

$$
\liminf _{\varepsilon \rightarrow 0} C_{\varepsilon}^{i} \geq E_{m_{i}} .
$$

Proof. Arguing by contradiction, we assume that $\liminf _{\varepsilon \rightarrow 0} C_{\varepsilon}^{i}<E_{m_{i}}$. Then, there exists $\alpha>0, \varepsilon_{n} \rightarrow 0$ and $\gamma_{n} \in \Phi_{\varepsilon_{n}}^{i}$ satisfying $\Gamma_{\varepsilon_{n}}^{i}\left(\gamma_{n}(s)\right)<E_{m_{i}}-\alpha$ for $s \in\left(0, T_{i}\right)$.

We fix an $\varepsilon_{n}>0$ such that

$$
\frac{m_{i}}{2} \varepsilon_{n}^{\mu}\left(1+\left(1+E_{m_{i}}\right)^{2 /(p+2)}\right)<\min \{\alpha, 1\}
$$

and $\mathcal{F}_{\varepsilon_{n}}\left(\gamma_{n}\left(T_{i}\right)\right)<-2$ and denote $\varepsilon_{n}$ by $\varepsilon$ and $\gamma_{n}$ by $\gamma$. 
Since $\mathcal{F}_{\varepsilon}(\gamma(0))=0$ we can find $s_{0} \in(0,1)$ such that $\mathcal{F}_{\varepsilon}(\gamma(s)) \geq-1$ for $s \in\left[0, s_{0}\right]$ and $\mathcal{F}_{\varepsilon}\left(\gamma\left(s_{0}\right)\right)=-1$. Then for any $s \in\left[0, s_{0}\right]$ we have

$$
Q_{\varepsilon}^{i}(\gamma(s)) \leq \Gamma_{\varepsilon}^{i}(\gamma(s))+1 \leq E_{m_{i}}-\alpha+1
$$

so that

$$
\int_{\mathbb{R}^{N} \backslash O_{\varepsilon}^{i}}|\gamma(s)|^{2} \mathrm{~d} y \leq \varepsilon^{6 / \mu}\left(1+\left(1+E_{m_{i}}\right)^{2 /(p+2)}\right) \quad \forall s \in\left[0, s_{0}\right] .
$$

Now we notice that for any $s \in\left[0, T_{i}\right],|\gamma(s)| \in H^{1}\left(\mathbb{R}^{N}, \mathbb{R}\right)$ and by the diamagnetic inequality (2.3)

$$
\int_{\mathbb{R}^{N}}|\nabla| \gamma(s)||^{2} \mathrm{~d} y \leq \int_{\mathbb{R}^{N}}\left|D^{\varepsilon} \gamma(s)\right|^{2} \mathrm{~d} y .
$$

Then by (3.10) we have that for $s \in\left[0, s_{0}\right]$

$$
\begin{aligned}
\mathcal{F}_{\varepsilon}(\gamma(s))= & \frac{1}{2} \int_{\mathbb{R}^{N}}\left|D^{\varepsilon} \gamma(s)\right|^{2} \mathrm{~d} y+\frac{m_{i}}{2} \int_{\mathbb{R}^{N}}|\gamma(s)|^{2} \mathrm{~d} y-\int_{\mathbb{R}^{N}} F\left(|\gamma(s)|^{2}\right) \mathrm{d} y \\
& +\frac{1}{2} \int_{\mathbb{R}^{N}}\left(V_{\varepsilon}(y)-m_{i}\right)|\gamma(s)|^{2} \mathrm{~d} y \\
\geq & \frac{1}{2} \int_{\mathbb{R}^{N}}|\nabla| \gamma(s)||^{2} \mathrm{~d} y+\frac{m_{i}}{2} \int_{\mathbb{R}^{N}}|\gamma(s)|^{2} \mathrm{~d} y-\int_{\mathbb{R}^{N}} F\left(|\gamma(s)|^{2}\right) \mathrm{d} y \\
& +\frac{1}{2} \int_{\mathbb{R}^{N} \backslash O_{\varepsilon}^{i}}\left(V_{\varepsilon}(y)-m_{i}\right)|\gamma(s)|^{2} \mathrm{~d} y \\
\geq & \left.\frac{1}{2} \int_{\mathbb{R}^{N}}|\nabla| \gamma(s)\right|^{2} \mathrm{~d} y+\frac{m_{i}}{2} \int_{\mathbb{R}^{N}}|\gamma(s)|^{2} \mathrm{~d} y-\int_{\mathbb{R}^{N}} F\left(|\gamma(s)|^{2}\right) \mathrm{d} y \\
& -\frac{m_{i}}{2} \int_{\mathbb{R}^{N} \backslash O_{\varepsilon}^{i}}|\gamma(s)|^{2} \mathrm{~d} y \\
\geq & \left.\frac{1}{2} \int_{\mathbb{R}^{N}}|\nabla| \gamma(s)\right|^{2} \mathrm{~d} y+\frac{m_{i}}{2} \int_{\mathbb{R}^{N}}|\gamma(s)|^{2} \mathrm{~d} y-\int_{\mathbb{R}^{N}} F\left(|\gamma(s)|^{2}\right) \mathrm{d} y \\
& -\frac{m_{i}}{2} \varepsilon^{6 / \mu}\left(1+\left(1+E_{m_{i}}\right)^{2 /(p+2)}\right) \\
= & L_{m_{i}}(|\gamma(s)|)-\frac{m_{i}}{2} \varepsilon^{6 / \mu}\left(1+\left(1+E_{m_{i}}\right)^{2 /(p+2)}\right) .
\end{aligned}
$$

Thus, $L_{m_{i}}\left(\left|\gamma\left(s_{0}\right)\right|\right)<0$ and recalling that for the limiting equation (2.7) the mountain pass level corresponds to the least energy level (see [32]) we have that

$$
\max _{s \in\left[0, T_{i}\right]} L_{m_{i}}(|\gamma(s)|) \geq E_{m_{i}}
$$

Then we infer that

$$
\begin{aligned}
E_{m_{i}}-\alpha & \geq \max _{s \in\left[0, T_{i}\right]} \Gamma_{\varepsilon}^{i}(\gamma(s)) \geq \max _{s \in\left[0, T_{i}\right]} \mathcal{F}_{\varepsilon}(\gamma(s)) \\
& \geq \max _{s \in\left[0, s_{0}\right]} \mathcal{F}_{\varepsilon}(\gamma(s)) \\
& \geq \max _{s \in\left[0, s_{0}\right]} L_{m_{i}}(|\gamma(s)|)-\frac{m_{i}}{2} \varepsilon^{6 / \mu}\left(1+\left(1+E_{m_{i}}\right)^{2 /(p+2)}\right) \\
& \geq E_{m_{i}}-\frac{m_{i}}{2} \varepsilon^{6 / \mu}\left(1+\left(1+E_{m_{i}}\right)^{2 /(p+2)}\right)
\end{aligned}
$$

and this contradiction completes the proof. 
Now we define

$$
\Gamma_{\varepsilon}^{\alpha}=\left\{u \in H_{\varepsilon} \mid \Gamma_{\varepsilon}(u) \leq \alpha\right\}
$$

Proposition 3.3. Let $\left(\varepsilon_{j}\right)$ be such that $\lim _{j \rightarrow \infty} \varepsilon_{j}=0$ and $\left(u_{\varepsilon_{j}}\right) \in X_{\varepsilon_{j}}^{d}$ such that

$$
\lim _{j \rightarrow \infty} \Gamma_{\varepsilon_{j}}\left(u_{\varepsilon_{j}}\right) \leq E \text { and } \lim _{j \rightarrow \infty} \Gamma_{\varepsilon_{j}}^{\prime}\left(u_{\varepsilon_{j}}\right)=0
$$

Then, for sufficiently small $d>0$, there exist, up to a subsequence, $\left(y_{j}^{i}\right) \subset \mathbb{R}^{N}, i=1, \ldots, k$, points $x^{i} \in \mathcal{M}^{i}$ (which should not be confused with the points $x_{i}$ already introduced), $U_{i} \in S_{m_{i}}$ such that

$$
\lim _{j \rightarrow \infty}\left|\varepsilon_{j} y_{j}^{i}-x^{i}\right|=0 \text { and } \lim _{j \rightarrow \infty}\left\|u_{\varepsilon_{j}}-\sum_{i=1}^{k} \mathrm{e}^{i A_{\varepsilon}\left(y_{j}^{i}\right)\left(\cdot-y_{j}^{i}\right)} \varphi_{\varepsilon_{j}}\left(\cdot-y_{j}^{i}\right) U_{i}\left(\cdot-y_{j}^{i}\right)\right\|_{\varepsilon_{j}}=0
$$

Proof. For simplicity we write $\varepsilon$ for $\varepsilon_{j}$. From Proposition 2.5, we know that the $S_{m_{i}}$ are compact. Then there exist $Z_{i} \in S_{m_{i}}$ and $\left(x_{\varepsilon}^{i}\right) \subset\left(\mathcal{M}^{i}\right)^{\beta}, x^{i} \in\left(\mathcal{M}^{i}\right)^{\beta}$ for $i=1, \ldots, k$, with $x_{\varepsilon}^{i} \rightarrow x^{i}$ as $\varepsilon \rightarrow 0$ such that, passing to a subsequence still denoted $\left(u_{\varepsilon}\right)$,

$$
\left\|u_{\varepsilon}-\sum_{i=1}^{k} \mathrm{e}^{i A\left(x_{\varepsilon}^{i}\right)\left(\cdot-\frac{x_{\varepsilon}^{i}}{\varepsilon}\right)} \varphi_{\varepsilon}\left(\cdot-x_{\varepsilon}^{i} / \varepsilon\right) Z_{i}\left(\cdot-x_{\varepsilon}^{i} / \varepsilon\right)\right\|_{\varepsilon} \leq 2 d
$$

for small $\varepsilon>0$. We set $u_{1, \varepsilon}=\sum_{i=1}^{k} \varphi_{\varepsilon}\left(\cdot-x_{\varepsilon}^{i} / \varepsilon\right) u_{\varepsilon}$ and $u_{2, \varepsilon}=u_{\varepsilon}-u_{1, \varepsilon}$. As a first step in the proof of the proposition we shall prove that

$$
\Gamma_{\varepsilon}\left(u_{\varepsilon}\right) \geq \Gamma_{\varepsilon}\left(u_{1, \varepsilon}\right)+\Gamma_{\varepsilon}\left(u_{2, \varepsilon}\right)+O(\varepsilon) .
$$

Suppose there exist $y_{\varepsilon} \in \bigcup_{i=1}^{k} B\left(x_{\varepsilon}^{i} / \varepsilon, 2 \beta / \varepsilon\right) \backslash B\left(x_{\varepsilon}^{i} / \varepsilon, \beta / \varepsilon\right)$ and $R>0$ satisfying

$$
\liminf _{\varepsilon \rightarrow 0} \int_{B\left(y_{\varepsilon}, R\right)}\left|u_{\varepsilon}\right|^{2} \mathrm{~d} y>0
$$

which means that

$$
\liminf _{\varepsilon \rightarrow 0} \int_{B(0, R)}\left|v_{\varepsilon}\right|^{2} \mathrm{~d} y>0
$$

where $v_{\varepsilon}(y)=u_{\varepsilon}\left(y+y_{\varepsilon}\right)$. Taking a subsequence, we can assume that $\varepsilon y_{\varepsilon} \rightarrow x_{0}$ with $x_{0}$ in the closure of $\bigcup_{i=1}^{k} B\left(x^{i}, 2 \beta\right) \backslash B\left(x^{i}, \beta\right)$. Since $(3.15)$ holds, $\left(v_{\varepsilon}\right)$ is bounded in $H_{\varepsilon}$. Thus, since $\tilde{m}>0,\left(v_{\varepsilon}\right)$ is bounded in $L^{2}\left(\mathbb{R}^{N}, \mathbb{C}\right)$ and using the diamagnetic inequality $(2.3)$ we deduce that $\left(v_{\varepsilon}\right)$ is bounded in $L^{p+2}\left(\mathbb{R}^{N}, \mathbb{C}\right)$. In particular, up to a subsequence, $v_{\varepsilon} \rightarrow W \in L^{p+2}\left(\mathbb{R}^{N}, \mathbb{C}\right)$ weakly. Also by Corollary 2.2 (i), for any compact $K \subset \mathbb{R}^{N},\left(v_{\varepsilon}\right)$ is bounded in $H^{1}(K, \mathbb{C})$. Thus we can assume that $v_{\varepsilon} \rightarrow W$ in $H^{1}(K, \mathbb{C})$ weakly for any $K \subset \mathbb{R}^{N}$ compact, strongly in $L^{p+2}(K, \mathbb{C})$. Because of $(3.17) W$ is not the zero function. Now, $\operatorname{since} \lim _{\varepsilon \rightarrow 0} \Gamma_{\varepsilon}^{\prime}\left(u_{\varepsilon}\right)=0$, $W$ is a non-trivial solution of

$$
-\Delta W-\frac{2}{i} A\left(x_{0}\right) \cdot \nabla W+\left|A\left(x_{0}\right)\right|^{2} W+V\left(x_{0}\right) W=f\left(|W|^{2}\right) W
$$

From $(3.18)$ and since $W \in L^{p+2}\left(\mathbb{R}^{N}, \mathbb{C}\right)$ we readily deduce, using Corollary 2.2 (ii) that $W \in H^{1}\left(\mathbb{R}^{N}, \mathbb{C}\right)$.

Let $\omega(y)=\mathrm{e}^{-i A\left(x_{0}\right) y} W(y)$. Then $\omega$ is a non trivial solution of the complex-valued equation

$$
-\Delta \omega+V\left(x_{0}\right) \omega(y)=f\left(|\omega|^{2}\right) \omega .
$$


For $R>0$ large we have

$$
\int_{B(0, R)}\left|\left(\frac{\nabla}{i}-A\left(x_{0}\right)\right) W\right|^{2} \mathrm{~d} y \geq \frac{1}{2} \int_{\mathbb{R}^{N}}\left|\left(\frac{\nabla}{i}-A\left(x_{0}\right)\right) W\right|^{2} \mathrm{~d} y
$$

and thus, by the weak convergence,

$$
\begin{aligned}
\liminf _{\varepsilon \rightarrow 0} \int_{B\left(y_{\varepsilon}, R\right)}\left|D^{\varepsilon} u_{\varepsilon}\right|^{2} \mathrm{~d} y & =\liminf _{\varepsilon \rightarrow 0} \int_{B(0, R)}\left|\left(\frac{\nabla}{i}-A_{\varepsilon}\left(y+y_{\varepsilon}\right)\right) v_{\varepsilon}\right|^{2} \mathrm{~d} y \\
& \geq \int_{B(0, R)}\left|\left(\frac{\nabla}{i}-A\left(x_{0}\right)\right) W\right|^{2} \mathrm{~d} y \\
& \geq \frac{1}{2} \int_{\mathbb{R}^{N}}\left|\left(\frac{\nabla}{i}-A\left(x_{0}\right)\right) W\right|^{2} \mathrm{~d} y=\frac{1}{2} \int_{\mathbb{R}^{N}}|\nabla \omega|^{2} \mathrm{~d} y .
\end{aligned}
$$

Now recalling from [32] that $E_{a}>E_{b}$ if $a>b$ and using Lemma 2.3 we have $L_{V\left(x_{0}\right)}^{c}(\omega) \geq E_{V\left(x_{0}\right)}^{c}=E_{V\left(x_{0}\right)} \geq E_{m}$ since $V\left(x_{0}\right) \geq m$. Thus from (3.20) and (2.10) we get that

$$
\liminf _{\varepsilon \rightarrow 0} \int_{B\left(y_{\varepsilon}, R\right)}\left|D^{\varepsilon} u_{\varepsilon}\right|^{2} \mathrm{~d} y \geq \frac{N}{2} L_{V\left(x_{0}\right)}^{c}(\omega) \geq \frac{N}{2} E_{m}>0,
$$

which contradicts (3.15), provided $d>0$ is small enough. Indeed, $x_{0} \neq x^{i}, \forall i \in\{1, \ldots, k\}$ and the $Z_{i}$ are exponentially decreasing.

Since such a sequence $\left(y_{\varepsilon}\right)$ does not exist, we deduce from [37], Lemma I.1, that

$$
\limsup _{\varepsilon \rightarrow 0} \int_{\bigcup_{i=1}^{k} B\left(x_{\varepsilon}^{i} / \varepsilon, 2 \beta / \varepsilon\right) \backslash B\left(x_{\varepsilon}^{i} / \varepsilon, \beta / \varepsilon\right)}\left|u_{\varepsilon}\right|^{p+2} \mathrm{~d} y=0 .
$$

As a consequence, we can derive using (f1), (f2) and the boundedness of $\left(\left\|u_{\varepsilon}\right\|_{2}\right)$ that

$$
\lim _{\varepsilon \rightarrow 0} \int_{\mathbb{R}^{N}} F\left(\left|u_{\varepsilon}\right|^{2}\right)-F\left(\left|u_{1, \varepsilon}\right|^{2}\right)-F\left(\left|u_{2, \varepsilon}\right|^{2}\right) \mathrm{d} y=0
$$

At this point writing

$$
\begin{aligned}
\Gamma_{\varepsilon}\left(u_{\varepsilon}\right)=\Gamma_{\varepsilon}\left(u_{1, \varepsilon}\right) & +\Gamma_{\varepsilon}\left(u_{2, \varepsilon}\right)+\sum_{i=1}^{k} \int_{B\left(x_{\varepsilon}^{i} / \varepsilon, 2 \beta / \varepsilon\right) \backslash B\left(x_{\varepsilon}^{i} / \varepsilon, \beta / \varepsilon\right)} \varphi_{\varepsilon}\left(y-x_{\varepsilon}^{i} / \varepsilon\right)\left(1-\varphi_{\varepsilon}\left(y-x_{\varepsilon}^{i} / \varepsilon\right)\right)\left|D^{\varepsilon} u_{\varepsilon}\right|^{2} \\
& +V_{\varepsilon} \varphi_{\varepsilon}\left(y-x_{\varepsilon}^{i} / \varepsilon\right)\left(1-\varphi_{\varepsilon}\left(y-x_{\varepsilon}^{i} / \varepsilon\right)\right)\left|u_{\varepsilon}\right|^{2} \mathrm{~d} y-\int_{\mathbb{R}^{N}} F\left(\left|u_{\varepsilon}\right|^{2}\right)-F\left(\left|u_{1, \varepsilon}\right|^{2}\right)-F\left(\left|u_{2, \varepsilon}\right|^{2}\right) \mathrm{d} y+o(1),
\end{aligned}
$$

as $\varepsilon \rightarrow 0$ this shows that the inequality (3.16) holds. We now estimate $\Gamma_{\varepsilon}\left(u_{2, \varepsilon}\right)$. We have

$$
\begin{aligned}
\Gamma_{\varepsilon}\left(u_{2, \varepsilon}\right) \geq \mathcal{F}_{\varepsilon}\left(u_{2, \varepsilon}\right) & =\frac{1}{2} \int_{\mathbb{R}^{N}}\left|D^{\varepsilon} u_{2, \varepsilon}\right|^{2}+\tilde{V}_{\varepsilon}\left|u_{2, \varepsilon}\right|^{2} \mathrm{~d} y-\frac{1}{2} \int_{\mathbb{R}^{N}}\left(\tilde{V}_{\varepsilon}-V_{\varepsilon}\right)\left|u_{2, \varepsilon}\right|^{2} \mathrm{~d} y-\int_{\mathbb{R}^{N}} F\left(\left|u_{2, \varepsilon}\right|^{2}\right) \mathrm{d} y \\
& \geq \frac{1}{2}\left\|u_{2, \varepsilon}\right\|_{\varepsilon}^{2}-\frac{\tilde{m}}{2} \int_{\mathbb{R}^{N} \backslash O_{\varepsilon}^{i}}\left|u_{2, \varepsilon}\right|^{2} \mathrm{~d} y-\int_{\mathbb{R}^{N}} F\left(\left|u_{2, \varepsilon}\right|^{2}\right) \mathrm{d} y
\end{aligned}
$$


Here we have used the fact that $\tilde{V}_{\varepsilon}-V_{\varepsilon}=0$ on $O_{\varepsilon}^{i}$ and $\left|\tilde{V}_{\varepsilon}-V_{\varepsilon}\right| \leq \widetilde{m}$ on $\mathbb{R}^{N} \backslash O_{\varepsilon}^{i}$. Because of (f1), (f2) for some $C_{1}, C_{2}>0$,

$$
\int_{\mathbb{R}^{N}} F\left(\left|u_{2, \varepsilon}\right|^{2}\right) \mathrm{d} y \leq \frac{\tilde{m}}{4} \int_{\mathbb{R}^{N}}\left|u_{2, \varepsilon}\right|^{2} \mathrm{~d} y+C_{1} \int_{\mathbb{R}^{N}}\left|u_{2, \varepsilon}\right|^{\frac{2 N}{N-2}} \mathrm{~d} y \leq \frac{\tilde{m}}{4} \int_{\mathbb{R}^{N}}\left|u_{2, \varepsilon}\right|^{2} \mathrm{~d} y+C_{2} \|\left. u_{2, \varepsilon}\right|_{\varepsilon} ^{\frac{2 N}{N-2}}
$$

Since $\left(u_{\varepsilon}\right)$ is bounded, we see from (3.15) that $\left\|u_{2, \varepsilon}\right\|_{\varepsilon} \leq 4 d$ for small $\varepsilon>0$. Thus taking $d>0$ small enough we have

$$
\frac{1}{2}\left\|u_{2, \varepsilon}\right\|_{\varepsilon}^{2}-\int_{\mathbb{R}^{N}} F\left(\left|u_{2, \varepsilon}\right|^{2}\right) \mathrm{d} y \geq\left\|u_{2, \varepsilon}\right\|_{\varepsilon}^{2}\left(\frac{1}{4}-C_{2}(4 d)^{4 /(N-2)}\right) \geq \frac{1}{8}\left\|u_{2, \varepsilon}\right\|_{\varepsilon}^{2} .
$$

Now note that $\mathcal{F}_{\varepsilon}$ is uniformly bounded in $X_{\varepsilon}^{d}$ for small $\varepsilon>0$. Thus, so is $Q_{\varepsilon}$. This implies that for some $C>0$,

$$
\int_{\mathbb{R}^{N} \backslash O_{\varepsilon}}\left|u_{2, \varepsilon}\right|^{2} \mathrm{~d} y \leq C \varepsilon^{6 / \mu}
$$

and from (3.23)-(3.25) we deduce that $\Gamma_{\varepsilon}\left(u_{2, \varepsilon}\right) \geq o(1)$.

Now for $i=1, \ldots, k$, we define $u_{1, \varepsilon}^{i}(y)=u_{1, \varepsilon}(y)$ for $y \in O_{\varepsilon}^{i}, u_{1, \varepsilon}^{i}(y)=0$ for $y \notin O_{\varepsilon}^{i}$. Also we set $W_{\varepsilon}^{i}(y)=$ $u_{1, \varepsilon}^{i}\left(y+x_{\varepsilon}^{i} / \varepsilon\right)$. We fix an arbitrary $i \in\{1, \ldots, k\}$. Arguing as before, we can assume, up to a subsequence, that $W_{\varepsilon}^{i}$ converges weakly in $L^{p+2}\left(\mathbb{R}^{N}, \mathbb{C}\right)$ to a solution $W^{i} \in H^{1}\left(\mathbb{R}^{N}, \mathbb{C}\right)$ of

$$
-\Delta W^{i}-\frac{2}{i} A\left(x^{i}\right) \cdot \nabla W^{i}+\left|A\left(x^{i}\right)\right|^{2} W^{i}+V\left(x^{i}\right) W^{i}=f\left(\left|W^{i}\right|^{2}\right) W^{i}, \quad y \in \mathbb{R}^{N} .
$$

We shall prove that $W_{\varepsilon}^{i}$ tends to $W^{i}$ strongly in $H_{\varepsilon}$. Suppose there exist $R>0$ and a sequence $\left(z_{\varepsilon}\right)$ with $z_{\varepsilon} \in B\left(x_{\varepsilon}^{i} / \varepsilon, 2 \beta / \varepsilon\right)$ satisfying

$$
\liminf _{\varepsilon \rightarrow 0}\left|z_{\varepsilon}-x_{\varepsilon}^{i} / \varepsilon\right|=\infty \quad \text { and } \quad \liminf _{\varepsilon \rightarrow 0} \int_{B\left(z_{\varepsilon}, R\right)}\left|u_{\varepsilon}^{1, i}\right|^{2} \mathrm{~d} y>0
$$

We may assume that $\varepsilon z_{\varepsilon} \rightarrow z^{i} \in O^{i}$ as $\varepsilon \rightarrow 0$. Then $\tilde{W}_{\varepsilon}^{i}(y)=W_{\varepsilon}^{i}\left(y+z_{\varepsilon}\right)$ weakly converges in $L^{p+2}\left(\mathbb{R}^{N}, \mathbb{C}\right)$ to $\tilde{W}^{i} \in H^{1}\left(\mathbb{R}^{N}, \mathbb{C}\right)$ which satisfies

$$
-\Delta \tilde{W}^{i}-\frac{2}{i} A\left(z^{i}\right) \cdot \nabla \tilde{W}+\left|A\left(z^{i}\right)\right|^{2} \tilde{W}^{i}+V\left(z^{i}\right) \tilde{W}^{i}=f\left(\left|\tilde{W}^{i}\right|^{2}\right) \tilde{W}^{i}, \quad y \in \mathbb{R}^{N}
$$

and as before we get a contradiction. Then using (f1), (f2) and [37], Lemma I.1, it follows that

$$
\int_{\mathbb{R}^{N}} F\left(\left|W_{\varepsilon}^{i}\right|^{2}\right) \mathrm{d} y \rightarrow \int_{\mathbb{R}^{N}} F\left(\left|W^{i}\right|^{2}\right) \mathrm{d} y
$$


Then from the weak convergence of $W_{\varepsilon}^{i}$ to $W^{i} \neq 0$ in $H^{1}(K, \mathbb{C})$ for any $K \subset \mathbb{R}^{N}$ compact we get, for any $i \in\{1, \ldots, k\}$,

$$
\begin{aligned}
\limsup _{\varepsilon \rightarrow 0} \Gamma_{\varepsilon}\left(u_{1, \varepsilon}^{i}\right) \geq & \liminf _{\varepsilon \rightarrow 0} \mathcal{F}_{\varepsilon}\left(u_{1, \varepsilon}^{i}\right) \\
\geq & \liminf _{\varepsilon \rightarrow 0} \frac{1}{2} \int_{B(0, R)}\left|\left(\frac{\nabla}{i}-A\left(\varepsilon y+x_{\varepsilon}^{i}\right)\right) W_{\varepsilon}^{i}\right|^{2} \\
& +V\left(\varepsilon y+x_{\varepsilon}^{i}\right)\left|W_{\varepsilon}^{i}\right|^{2} \mathrm{~d} y-\int_{\mathbb{R}^{N}} F\left(\left|W_{\varepsilon}^{i}\right|^{2}\right) \mathrm{d} y \\
\geq & \frac{1}{2} \int_{B(0, R)}\left|\left(\frac{\nabla}{i}-A\left(x^{i}\right)\right) W^{i}\right|^{2}+V\left(x^{i}\right)\left|W^{i}\right|^{2} \mathrm{~d} y \\
& -\int_{\mathbb{R}^{N}} F\left(\left|W^{i}\right|^{2}\right) \mathrm{d} y .
\end{aligned}
$$

Since these inequalities hold for any $R>0$ we deduce, using Lemma 2.3, that

$$
\begin{aligned}
\limsup _{\varepsilon \rightarrow 0} \Gamma_{\varepsilon}\left(u_{1, \varepsilon}^{i}\right) \geq & \frac{1}{2} \int_{\mathbb{R}^{N}}\left|\left(\frac{\nabla}{i}-A\left(x^{i}\right)\right) W^{i}\right|^{2} \mathrm{~d} y+\frac{1}{2} \int_{\mathbb{R}^{N}} V\left(x^{i}\right)\left|W^{i}\right|^{2} \mathrm{~d} y \\
& -\int_{\mathbb{R}^{N}} F\left(\left|W^{i}\right|^{2}\right) \mathrm{d} y \\
= & \frac{1}{2} \int_{\mathbb{R}^{N}}\left|\nabla \omega^{i}\right|^{2}+V\left(x^{i}\right)\left|\omega^{i}\right|^{2} \mathrm{~d} y-\int_{\mathbb{R}^{N}} F\left(\left|\omega^{i}\right|^{2}\right) \mathrm{d} y \\
= & L_{V\left(x^{i}\right)}^{c}\left(\omega^{i}\right) \geq E_{m_{i}}^{c}=E_{m_{i}}
\end{aligned}
$$

where we have set $\omega^{i}(y)=\mathrm{e}^{-i A\left(x^{i}\right) y} W^{i}(y)$. Now by (3.16),

$$
\limsup _{\varepsilon \rightarrow 0}\left(\Gamma_{\varepsilon}\left(u_{2, \varepsilon}\right)+\sum_{i=1}^{k} \Gamma_{\varepsilon}\left(u_{1, \varepsilon}^{i}\right)\right)=\limsup _{\varepsilon \rightarrow 0}\left(\Gamma_{\varepsilon}\left(u_{2, \varepsilon}\right)+\Gamma_{\varepsilon}\left(u_{1, \varepsilon}\right)\right) \leq \limsup _{\varepsilon \rightarrow 0} \Gamma_{\varepsilon}\left(u_{\varepsilon}\right) \leq E=\sum_{i=1}^{k} E_{m_{i}}
$$

Thus, since $\Gamma_{\varepsilon}\left(u_{2, \varepsilon}\right) \geq o(1)$ we deduce from (3.28)-(3.29) that, for any $i \in\{1, \ldots k\}$

$$
\lim _{\varepsilon \rightarrow 0} \Gamma_{\varepsilon}\left(u_{1, \varepsilon}^{i}\right)=E_{m_{i}}
$$

Now (3.28), (3.30) implies that $L_{V\left(x^{i}\right)}\left(\omega^{i}\right)=E_{m_{i}}$. Recalling from [32] that $E_{a}>E_{b}$ if $a>b$ and using Lemma 2.3 we conclude that $x^{i} \in \mathcal{M}^{i}$. At this point it is clear that $W^{i}(y)=\mathrm{e}^{i A\left(x^{i}\right) y} U_{i}\left(y-z_{i}\right)$ with $U_{i} \in S_{m_{i}}$ and $z_{i} \in \mathbb{R}^{N}$.

To establish that $W_{\varepsilon}^{i} \rightarrow W^{i}$ strongly in $H_{\varepsilon}$ we first show that $W_{\varepsilon}^{i} \rightarrow W^{i}$ strongly in $L^{2}\left(\mathbb{R}^{N}, \mathbb{C}\right)$. Since $\left(W_{\varepsilon}^{i}\right)$ is bounded in $H_{\varepsilon}$ the diamagnetic inequality (2.3) immediately yields that $\left(\left|W_{\varepsilon}^{i}\right|\right)$ is bounded in $H^{1}\left(\mathbb{R}^{N}, \mathbb{R}\right)$ and we can assume that $\left|W_{\varepsilon}^{i}\right| \rightarrow\left|W^{i}\right|=\left|\omega^{i}\right|$ weakly in $H^{1}\left(\mathbb{R}^{N}, \mathbb{R}\right)$. Now since $L_{V\left(x^{i}\right)}\left(\omega^{i}\right)=E_{m_{i}}$, we get using the 
diamagnetic inequality, (3.26), (3.30) and the fact that $V \geq V\left(x^{i}\right)$ on $O^{i}$,

$$
\begin{aligned}
\int_{\mathbb{R}^{N}}\left|\nabla \omega^{i}\right|^{2} \mathrm{~d} y+ & \int_{\mathbb{R}^{N}} m_{i}\left|\omega^{i}\right|^{2} \mathrm{~d} y-2 \int_{\mathbb{R}^{N}} F\left(\left|\omega^{i}\right|^{2}\right) \mathrm{d} y \geq \\
& \limsup _{\varepsilon \rightarrow 0} \int_{\mathbb{R}^{N}}\left|\left(\frac{\nabla}{i}-A\left(\varepsilon y+x_{\varepsilon}^{i}\right)\right) W_{\varepsilon}^{i}\right|^{2} \mathrm{~d} y+\int_{\mathbb{R}^{N}} V\left(\varepsilon y+x_{\varepsilon}^{i}\right)\left|W_{\varepsilon}^{i}\right|^{2} \mathrm{~d} y \\
& \quad-2 \int_{\mathbb{R}^{N}} F\left(\left|W_{\varepsilon}^{i}\right|^{2}\right) \mathrm{d} y \\
\geq & \left.\limsup _{\varepsilon \rightarrow 0} \int_{\mathbb{R}^{N}}|\nabla| W_{\varepsilon}^{i}\right|^{2} \mathrm{~d} y+\int_{\mathbb{R}^{N}} V\left(x^{i}\right)\left|W_{\varepsilon}^{i}\right|^{2} \mathrm{~d} y-2 \int_{\mathbb{R}^{N}} F\left(\left|W_{\varepsilon}^{i}\right|^{2}\right) \mathrm{d} y \\
\geq & \int_{\mathbb{R}^{N}}|\nabla| \omega^{i}||^{2} \mathrm{~d} y+\int_{\mathbb{R}^{N}} m_{i}\left|\omega^{i}\right|^{2} \mathrm{~d} y-2 \int_{\mathbb{R}^{N}} F\left(\left|\omega^{i}\right|^{2}\right) \mathrm{d} y .
\end{aligned}
$$

But from Lemma 2.3 we know that, since $L_{V\left(x^{i}\right)}\left(\omega^{i}\right)=E_{m_{i}}$,

$$
\int_{\mathbb{R}^{N}}|\nabla| \omega^{i}||^{2} \mathrm{~d} y=\int_{\mathbb{R}^{N}}\left|\nabla \omega^{i}\right|^{2} \mathrm{~d} y
$$

Thus we deduce from (3.31) that

$$
\int_{\mathbb{R}^{N}} V\left(\varepsilon y+x_{\varepsilon}^{i}\right)\left|W_{\varepsilon}^{i}\right|^{2} \mathrm{~d} y \rightarrow \int_{\mathbb{R}^{N}} V\left(x^{i}\right)\left|W^{i}\right|^{2} \mathrm{~d} y .
$$

Thus, since $V \geq V\left(x^{i}\right)$ on $O^{i}$, we deduce that

$$
W_{\varepsilon}^{i} \rightarrow W^{i} \text { strongly in } L^{2}\left(\mathbb{R}^{N}, \mathbb{C}\right) .
$$

From (3.33) we easily get that

$$
\lim _{\varepsilon \rightarrow 0} \int_{\mathbb{R}^{N}}\left|\left(\frac{\nabla}{i}-A\left(\varepsilon y+x_{\varepsilon}^{i}\right)\right) W_{\varepsilon}^{i}\right|^{2}-\left|\left(\frac{\nabla}{i}-A\left(x^{i}\right)\right) W_{\varepsilon}^{i}\right|^{2} \mathrm{~d} y=0 .
$$

Now, using (3.26), (3.31) and (3.32), we see from (3.34) that

$$
\begin{aligned}
& \int_{\mathbb{R}^{N}}\left|\left(\frac{\nabla}{i}-A\left(x^{i}\right)\right) W^{i}\right|^{2} \mathrm{~d} y+\int_{\mathbb{R}^{N}} V\left(x^{i}\right)\left|W^{i}\right|^{2} \mathrm{~d} y \geq \\
& \limsup _{\varepsilon \rightarrow 0} \int_{\mathbb{R}^{N}}\left|\left(\frac{\nabla}{i}-A\left(\varepsilon y+x_{\varepsilon}^{i}\right)\right) W_{\varepsilon}^{i}\right|^{2} \mathrm{~d} y+\int_{\mathbb{R}^{N}} V\left(\varepsilon y+x_{\varepsilon}^{i}\right)\left|W_{\varepsilon}^{i}\right|^{2} \mathrm{~d} y \\
& \quad \geq \limsup _{\varepsilon \rightarrow 0} \int_{\mathbb{R}^{N}}\left|\left(\frac{\nabla}{i}-A\left(x^{i}\right)\right) W_{\varepsilon}^{i}\right|^{2} \mathrm{~d} y+\int_{\mathbb{R}^{N}} V\left(x^{i}\right)\left|W_{\varepsilon}^{i}\right|^{2} \mathrm{~d} y .
\end{aligned}
$$

At this point and using Corollary 2.2 (ii) we have established the strong convergence $W_{\varepsilon}^{i} \rightarrow W^{i}$ in $H^{1}\left(\mathbb{R}^{N}, \mathbb{C}\right)$. Thus we have

$$
u_{1, \varepsilon}^{i}=\mathrm{e}^{i A\left(x^{i}\right)\left(\cdot-x_{\varepsilon}^{i} / \varepsilon\right)} U_{i}\left(\cdot-x_{\varepsilon}^{i} / \varepsilon-z_{i}\right)+o(1)
$$

strongly in $H^{1}\left(\mathbb{R}^{N}, \mathbb{C}\right)$. Now setting $y_{\varepsilon}^{i}=x_{\varepsilon}^{i} / \varepsilon+z_{i}$ and changing $U_{i}$ to $\mathrm{e}^{i A\left(x^{i}\right) z_{i}} U_{i}$ we get that

$$
u_{1, \varepsilon}^{i}=\mathrm{e}^{i A\left(x^{i}\right)\left(\cdot-y_{\varepsilon}^{i}\right)} U_{i}\left(\cdot-y_{\varepsilon}^{i}\right)+o(1)
$$


strongly in $H^{1}\left(\mathbb{R}^{N}, \mathbb{C}\right)$. Finally using the exponential decay of $U_{i}$ et $\nabla U_{i}$ we have

$$
u_{1, \varepsilon}^{i}=\mathrm{e}^{i A_{\varepsilon}\left(y_{\varepsilon}^{i}\right)\left(\cdot-y_{\varepsilon}^{i}\right)} \varphi_{\varepsilon}\left(\cdot-y_{\varepsilon}^{i}\right) U_{i}\left(\cdot-y_{\varepsilon}^{i}\right)+o(1) .
$$

From Corollary 2.2 (iii) we deduce that this convergence also holds in $H_{\varepsilon}$ and thus

$$
u_{1, \varepsilon}=\sum_{i=1}^{k} u_{1, \varepsilon}^{i}=\sum_{i=1}^{k} \mathrm{e}^{i A_{\varepsilon}\left(y_{\varepsilon}^{i}\right)\left(\cdot-y_{\varepsilon}^{i}\right)} \varphi_{\varepsilon}\left(\cdot-y_{\varepsilon}^{i}\right) U_{i}\left(\cdot-y_{\varepsilon}^{i}\right)+o(1)
$$

strongly in $H_{\varepsilon}$. To conclude the proof of the proposition, it suffices to show that $u_{2, \varepsilon} \rightarrow 0$ in $H_{\varepsilon}$. Since $E \geq \lim _{\varepsilon \rightarrow 0} \Gamma_{\varepsilon}\left(u_{\varepsilon}\right)$ and $\lim _{\varepsilon \rightarrow 0} \Gamma_{\varepsilon}\left(u_{1, \varepsilon}\right)=E$ we deduce, using (3.16) that $\lim _{\varepsilon \rightarrow 0} \Gamma_{\varepsilon}\left(u_{2, \varepsilon}\right)=0$. Now from (3.23)-(3.25) we get that $u_{2, \varepsilon} \rightarrow 0$ in $H_{\varepsilon}$.

Proposition 3.4. For sufficiently small $d>0$, there exist constants $\omega>0$ and $\varepsilon_{0}>0$ such that $\left|\Gamma_{\varepsilon}^{\prime}(u)\right| \geq \omega$ for $u \in \Gamma_{\varepsilon}^{D_{\varepsilon}} \cap\left(X_{\varepsilon}^{d} \backslash X_{\varepsilon}^{d / 2}\right)$ and $\varepsilon \in\left(0, \varepsilon_{0}\right)$.

Proof. By contradiction, we suppose that for $d>0$ sufficiently small such that Proposition 3.3 applies, there exist $\left(\varepsilon_{j}\right)$ with $\lim _{j \rightarrow \infty} \varepsilon_{j}=0$ and a sequence $\left(u_{\varepsilon_{j}}\right)$ with $u_{\varepsilon_{j}} \in X_{\varepsilon_{j}}^{d} \backslash X_{\varepsilon_{j}}^{d / 2}$ satisfying $\lim _{j \rightarrow \infty} \Gamma_{\varepsilon_{j}}\left(u_{\varepsilon_{j}}\right) \leq E$ and $\lim _{j \rightarrow \infty} \Gamma_{\varepsilon_{j}}^{\prime}\left(u_{\varepsilon_{j}}\right)=0$. By Proposition 3.3, there exist $\left(y_{\varepsilon_{j}}^{i}\right) \subset \mathbb{R}^{N}, i=1, \ldots, k, x^{i} \in \mathcal{M}^{i}, U_{i} \in S_{m_{i}}$ such that

$$
\begin{gathered}
\lim _{\varepsilon_{j} \rightarrow 0}\left|\varepsilon_{j} y_{\varepsilon_{j}}^{i}-x^{i}\right|=0 \\
\lim _{\varepsilon_{j} \rightarrow 0}\left\|u_{\varepsilon_{j}}-\sum_{i=1}^{k} \mathrm{e}^{i A_{\varepsilon_{j}}\left(y_{\varepsilon_{j}}^{i}\right)\left(\cdot-y_{\varepsilon_{j}}^{i}\right)} \varphi_{\varepsilon_{j}}\left(\cdot-y_{\varepsilon_{j}}^{i}\right) U_{i}\left(\cdot-y_{\varepsilon_{j}}^{i}\right)\right\|_{\varepsilon_{j}}=0 .
\end{gathered}
$$

By definition of $X_{\varepsilon_{j}}$ we see that $\lim _{\varepsilon_{j} \rightarrow 0} \operatorname{dist}\left(u_{\varepsilon_{j}}, X_{\varepsilon_{j}}\right)=0$. This contradicts that $u_{\varepsilon_{j}} \notin X_{\varepsilon_{j}}^{d / 2}$ and completes the proof.

From now on we fix a $d>0$ such that Proposition 3.4 holds.

Proposition 3.5. For sufficiently small fixed $\varepsilon>0, \Gamma_{\varepsilon}$ has a critical point $u_{\varepsilon} \in X_{\varepsilon}^{d} \cap \Gamma_{\varepsilon}^{D_{\varepsilon}}$.

Proof. We can take $R_{0}>0$ sufficiently large so that $O \subset B\left(0, R_{0}\right)$ and $\gamma_{\varepsilon}(s) \in H_{0}^{1}(B(0, R / \varepsilon))$ for any $s \in T$, $R>R_{0}$ and sufficiently small $\varepsilon>0$.

We notice that by Proposition 3.1 (iii), there exists $\alpha \in(0, E-\tilde{E})$ such that for sufficiently small $\varepsilon>0$,

$$
\Gamma_{\varepsilon}\left(\gamma_{\varepsilon}(s)\right) \geq D_{\varepsilon}-\alpha \quad \Longrightarrow \quad \gamma_{\varepsilon}(s) \in X_{\varepsilon}^{d / 2} \cap H_{0}^{1}(B(0, R / \varepsilon)) .
$$

We begin to show that for sufficiently small fixed $\varepsilon>0$, and $R>R_{0}$, there exists a sequence $\left(u_{n}^{R}\right) \subset X_{\varepsilon}^{d / 2} \cap$ $\Gamma_{\varepsilon}^{D_{\varepsilon}} \cap H_{0}^{1}(B(0, R / \varepsilon))$ such that $\Gamma^{\prime}\left(u_{n}^{R}\right) \rightarrow 0$ in $H_{0}^{1}(B(0, R / \varepsilon))$ as $n \rightarrow+\infty$.

Arguing by contradiction, we suppose that for sufficiently small $\varepsilon>0$, there exists $a_{R}(\varepsilon)>0$ such that $\left|\Gamma_{\varepsilon}^{\prime}(u)\right| \geq a_{R}(\varepsilon)$ on $X_{\varepsilon}^{d} \cap \Gamma_{\varepsilon}^{D_{\varepsilon}} \cap H_{0}^{1}(B(0, R / \varepsilon))$. In what follows any $u \in H_{0}^{1}(B(0, R / \varepsilon))$ will be regarded as an element in $H_{\varepsilon}$ by defining $u=0$ in $\mathbb{R}^{N} \backslash B(0, R / \varepsilon)$.

Note from Proposition 3.4 that there exists $\omega>0$, independent of $\varepsilon>0$, such that $\left|\Gamma_{\varepsilon}^{\prime}(u)\right| \geq \omega$ for $u \in$ $\Gamma_{\varepsilon}^{D_{\varepsilon}} \cap\left(X_{\varepsilon}^{d} \backslash X_{\varepsilon}^{d / 2}\right)$. Thus, by a deformation argument in $H_{0}^{1}(B(0, R / \varepsilon))$, starting from $\gamma_{\varepsilon}$, for sufficiently small $\varepsilon>0$ there exists a $\mu \in(0, \alpha)$ and a path $\gamma \in C\left([0, T], H_{\varepsilon}\right)$ satisfying

$$
\begin{gathered}
\gamma(s)=\gamma_{\varepsilon}(s) \quad \text { for } \gamma_{\varepsilon}(s) \in \Gamma_{\varepsilon}^{D_{\varepsilon}-\alpha}, \\
\gamma(s) \in X_{\varepsilon}^{d} \quad \text { for } \gamma_{\varepsilon}(s) \notin \Gamma_{\varepsilon}^{D_{\varepsilon}-\alpha}
\end{gathered}
$$


and

$$
\Gamma_{\varepsilon}(\gamma(s))<D_{\varepsilon}-\mu, \quad s \in T .
$$

Let $\psi \in C_{0}^{\infty}\left(\mathbb{R}^{N}\right)$ be such that $\psi(y)=1$ for $y \in O^{\delta}, \psi(y)=0$ for $y \notin O^{2 \delta}, \psi(y) \in[0,1]$ and $|\nabla \psi| \leq 2 / \delta$. For $\gamma(s) \in X_{\varepsilon}^{d}$, we define $\gamma_{1}(s)=\psi_{\varepsilon} \gamma(s)$ and $\gamma_{2}(s)=\left(1-\psi_{\varepsilon}\right) \gamma(s)$ where $\psi_{\varepsilon}(y)=\psi(\varepsilon y)$. Note that

$$
\begin{aligned}
\Gamma_{\varepsilon}(\gamma(s))=\Gamma_{\varepsilon}\left(\gamma_{1}(s)\right) & +\Gamma_{\varepsilon}\left(\gamma_{2}(s)\right)+\int_{\mathbb{R}^{N}}\left(\psi_{\varepsilon}\left(1-\psi_{\varepsilon}\right)\left|D^{\varepsilon} \gamma(s)\right|^{2}+V_{\varepsilon} \psi_{\varepsilon}\left(1-\psi_{\varepsilon}\right)|\gamma(s)|^{2}\right) \mathrm{d} y \\
& +Q_{\varepsilon}(\gamma(s))-Q_{\varepsilon}\left(\gamma_{1}(s)\right)-Q_{\varepsilon}\left(\gamma_{2}(s)\right)-\int_{\mathbb{R}^{N}}\left(F\left(|\gamma(s)|^{2}\right)-F\left(\left|\gamma_{1}(s)\right|^{2}\right)-F\left(\left|\gamma_{2}(s)\right|^{2}\right)\right) \mathrm{d} y+o(1)
\end{aligned}
$$

Since for $A, B \geq 0,(A+B-1)_{+} \geq(A-1)_{+}+(B-1)_{+}$and since $p+2 \geq 2$ it follows that

$$
\begin{aligned}
Q_{\varepsilon}(\gamma(s)) & =\left(\int_{\mathbb{R}^{N}} \chi_{\varepsilon}\left|\gamma_{1}(s)+\gamma_{2}(s)\right|^{2} \mathrm{~d} y-1\right)_{+}^{\frac{p+2}{2}} \\
& \geq\left(\int_{\mathbb{R}^{N}} \chi_{\varepsilon}\left|\gamma_{1}(s)\right|^{2} \mathrm{~d} y+\int_{\mathbb{R}^{N}} \chi_{\varepsilon}\left|\gamma_{2}(s)\right|^{2} \mathrm{~d} y-1\right)_{+}^{\frac{p+2}{2}} \\
& \geq\left(\int_{\mathbb{R}^{N}} \chi_{\varepsilon}\left|\gamma_{1}(s)\right|^{2} \mathrm{~d} y-1\right)_{+}^{\frac{p+2}{2}}+\left(\int_{\mathbb{R}^{N}} \chi_{\varepsilon}\left|\gamma_{2}(s)\right|^{2} \mathrm{~d} y-1\right)_{+}^{\frac{p+2}{2}} \\
& =Q_{\varepsilon}\left(\gamma_{1}(s)\right)+Q_{\varepsilon}\left(\gamma_{2}(s)\right)
\end{aligned}
$$

Now, as in the derivation of (3.25), using the fact that $Q_{\varepsilon}(\gamma(s))$ is uniformly bounded we have, for some $C>0$

$$
\int_{\mathbb{R}^{N} \backslash O_{\varepsilon}}|\gamma(s)|^{2} \mathrm{~d} y \leq C \varepsilon^{6 / \mu}
$$

Thus denoting $p+2=2 s+(1-s) \frac{2 N}{N-2}, s \in(0,1)$, we see from (f1), (f2), (3.37) and using the Sobolev inequalities, that for some $C_{1}, C_{2}>0$,

$$
\begin{aligned}
\int_{\mathbb{R}^{N} \backslash O_{\varepsilon}} F(\gamma(s)) \mathrm{d} y & \leq C_{1} \int_{\mathbb{R}^{N} \backslash O_{\varepsilon}}|\gamma(s)|^{2}+|\gamma(s)|^{p+2} \mathrm{~d} y \\
& \leq C_{1} \int_{\mathbb{R}^{N} \backslash O_{\varepsilon}}|\gamma(s)|^{2} \mathrm{~d} y \\
& +C_{2}\left(\int_{\mathbb{R}^{N} \backslash O_{\varepsilon}}|\gamma(s)|^{2} \mathrm{~d} y\right)^{s}\|\gamma(s)\|_{\varepsilon}^{(1-s) \frac{2 N}{N-2}}
\end{aligned}
$$

We deduce that

$$
\lim _{\varepsilon \rightarrow 0} \int_{\mathbb{R}^{N} \backslash O_{\varepsilon}} F(\gamma(s)) \mathrm{d} y=0 .
$$

Now, as $\varepsilon \rightarrow 0$

$$
\begin{aligned}
\int_{\mathbb{R}^{N}}\left|F(\gamma(s))-F\left(\gamma_{1}(s)\right)-F\left(\gamma_{2}(s)\right)\right| \mathrm{d} y & =\int_{\left(O^{2 \delta}\right)_{\varepsilon} \backslash\left(O^{\delta}\right)_{\varepsilon}}\left|F(\gamma(s))-F\left(\gamma_{1}(s)\right)-F\left(\gamma_{2}(s)\right)\right| \mathrm{d} y \\
& \leq \int_{\left(O^{2 \delta}\right)_{\varepsilon} \backslash\left(O^{\delta}\right)_{\varepsilon}} F(\gamma(s))+F\left(\gamma_{1}(s)\right)+F\left(\gamma_{2}(s)\right) \mathrm{d} y=o(1)
\end{aligned}
$$


since (3.39) obviously hold when $\gamma(s)$ is replaced by $\gamma_{1}(s)$ or $\gamma_{2}(s)$. Thus, we see that, as $\varepsilon \rightarrow 0$,

$$
\Gamma_{\varepsilon}(\gamma(s)) \geq \Gamma_{\varepsilon}\left(\gamma_{1}(s)\right)+\Gamma_{\varepsilon}\left(\gamma_{2}(s)\right)+o(1) .
$$

Also

Therefore it follows that

$$
\Gamma_{\varepsilon}\left(\gamma_{2}(s)\right) \geq-\int_{\mathbb{R}^{N} \backslash O_{\varepsilon}} F\left(\gamma_{2}(s)\right) \mathrm{d} y \geq o(1)
$$

$$
\Gamma_{\varepsilon}(\gamma(s)) \geq \Gamma_{\varepsilon}\left(\gamma_{1}(s)\right)+o(1) .
$$

For $i=1, \ldots, k$, we define $\gamma_{1}^{i}(s)(y)=\gamma_{1}(s)(y)$ for $y \in\left(O^{i}\right)_{\varepsilon}^{2 \delta}, \gamma_{1}^{i}(s)(y)=0$ for $y \notin\left(O^{i}\right)_{\varepsilon}^{2 \delta}$. Note that $\left(A_{1}+\ldots+A_{n}-1\right)_{+} \geq \sum_{i=1}^{n}\left(A_{i}-1\right)_{+}$for $A_{1}, \ldots, A_{n} \geq 0$, and that $(p+2) \geq 2$. Then, we see that,

$$
\Gamma_{\varepsilon}\left(\gamma_{1}(s)\right) \geq \sum_{i=1}^{k} \Gamma_{\varepsilon}\left(\gamma_{1}^{i}(s)\right)=\sum_{i=1}^{k} \Gamma_{\varepsilon}^{i}\left(\gamma_{1}^{i}(s)\right)
$$

From Proposition 3.1 (ii) and since $\alpha \in(0, E-\tilde{E})$ we get that $\gamma_{1}^{i} \in \Phi_{\varepsilon}^{i}$, for all $i \in\{1, \ldots, k\}$. Thus by Proposition 3.4 in [22], Proposition 3.2, and (3.41) we deduce that, as $\varepsilon \rightarrow 0$,

$$
\max _{s \in T} \Gamma_{\varepsilon}(\gamma(s)) \geq E+o(1)
$$

Since $\lim \sup _{\varepsilon \rightarrow 0} D_{\varepsilon} \leq E$ this contradicts (3.36).

Now let $\left(u_{n}^{R}\right)$ be a Palais-Smale sequence corresponding to a fixed small $\varepsilon>0$. Since $\left(u_{n}^{R}\right)$ is bounded in $H_{0}^{1}(B(0, R / \varepsilon))$, thanks to local compactness, we deduce that $\left(u_{n}^{R}\right)$ converges, up to subsequence, strongly to some $u^{R}$ in $H_{0}^{1}(B(0, R / \varepsilon))$ with $u^{R}$ a critical point of $\Gamma_{\varepsilon}$ on $H_{0}^{1}(B(0, R / \varepsilon))$.

Arguing as in Proposition 2 in [8], we now derive that $\left(u^{R}\right)$ converges strongly to some $u_{\varepsilon}$, as $R \rightarrow \infty$, with $u_{\varepsilon} \in X_{\varepsilon}^{d} \cap \Gamma_{\varepsilon}^{D_{\varepsilon}}$ a critical point of $\Gamma_{\varepsilon}$.

Indeed, since $u^{R}$ is a critical point of $\Gamma_{\varepsilon}$ on $H_{0}^{1}(B(0, R / \varepsilon))$ it satisfies

$$
\left(\frac{1}{i} \nabla-A_{\varepsilon}\right)^{2} u^{R}+V_{\varepsilon} u^{R}=f\left(\left|u^{R}\right|^{2}\right) u^{R}-(p+2)\left(\int \chi_{\varepsilon}\left|u^{R}\right|^{2} \mathrm{~d} y-1\right)_{+}^{\frac{p}{2}} \chi_{\varepsilon} u^{R} \text { in } B(0, R / \varepsilon) .
$$

Exploiting Kato's inequality (see [41], Thm. X.33)

$$
\Delta\left|u^{R}\right| \geq-\operatorname{Re}\left(\frac{\overline{u^{R}}}{\left|u^{R}\right|}\left(\frac{\nabla}{i}-A_{\varepsilon}(y)\right)^{2} u^{R}\right)
$$

we obtain

$$
\Delta\left|u^{R}\right| \geq V_{\varepsilon}\left|u^{R}\right|-f\left(\left|u^{R}\right|^{2}\right)\left|u^{R}\right|+(p+2)\left(\int \chi_{\varepsilon}\left|u^{R}\right|^{2} \mathrm{~d} y-1\right)_{+}^{\frac{p}{2}} \chi_{\varepsilon}\left|u^{R}\right| \text { in } \mathbb{R}^{N} .
$$

Moreover by Moser iteration [29] it follows that $\left(\left\|u^{R}\right\|_{L^{\infty}}\right)$ is bounded. Then, since $\left(Q_{\varepsilon}\left(u^{R}\right)\right)$ is uniformly bounded for small $\varepsilon>0$, we see from elliptic estimates that for sufficiently small $\varepsilon>0,\left|f\left(\left|u^{R}(y)\right|^{2}\right) u^{R}(y)\right| \leq$ $\frac{1}{2} V_{\varepsilon}\left|u^{R}(y)\right|$ if $|y| \geq 2 R_{0}$ (recall that $R_{0}>0$ can be chosen so large that $Z \subset B\left(0, R_{0}\right)$ ). Applying a comparison principle to (3.43), we see that for some $C, c>0$, independent of $R>R_{0}$,

$$
\left|u^{R}(y)\right| \leq C \exp \left(-\left(|y|-2 R_{0}\right)\right) .
$$

Now, observe that since $\left(u^{R}\right)$ is bounded in $H_{\varepsilon}$, we may assume that it converges weakly to some $u_{\varepsilon}$ in $H_{\varepsilon}$ as $R \rightarrow \infty$. Then, since $u^{R}$ is a solution of (3.42), we see from (3.44) that $\left(u^{R}\right)$ converges strongly to $u_{\varepsilon} \in X_{\varepsilon}^{d} \cap \Gamma_{\varepsilon}^{D_{\varepsilon}}$ 
and that

$$
\left(\frac{1}{i} \nabla-A_{\varepsilon}\right)^{2} u_{\varepsilon}+V_{\varepsilon} u_{\varepsilon}=f\left(\left|u_{\varepsilon}\right|^{2}\right) u_{\varepsilon}-(p+2)\left(\int \chi_{\varepsilon}\left|u_{\varepsilon}\right|^{2} \mathrm{~d} y-1\right)_{+}^{\frac{p}{2}} \chi_{\varepsilon} u_{\varepsilon} \text { in } \mathbb{R}^{N} .
$$

This ends the proof of the proposition.

Completion of the Proof for Theorem 1.1. We see from Proposition 3.5 that there exists $\varepsilon_{0}>0$ such that, for $\varepsilon \in\left(0, \varepsilon_{0}\right), \Gamma_{\varepsilon}$ has a critical point $u_{\varepsilon} \in X_{\varepsilon}^{d} \cap \Gamma_{\varepsilon}^{D_{\varepsilon}}$. From (3.45), as in the proof of Proposition 3.5, we obtain that

$$
\Delta\left|u_{\varepsilon}\right| \geq V_{\varepsilon}\left|u_{\varepsilon}\right|-f\left(\left|u_{\varepsilon}\right|^{2}\right)\left|u_{\varepsilon}\right|+(p+2)\left(\int \chi_{\varepsilon}\left|u_{\varepsilon}\right|^{2} \mathrm{~d} y-1\right)_{+}^{\frac{p}{2}} \chi_{\varepsilon}\left|u_{\varepsilon}\right| \text { in } \mathbb{R}^{N}
$$

and that $\left(\left\|u_{\varepsilon}\right\|_{L^{\infty}}\right)$ is bounded. Now by Proposition 3.3, we see that

$$
\lim _{\varepsilon \rightarrow 0} \int_{\mathbb{R}^{N} \backslash\left(\mathcal{M}^{2 \beta}\right)_{\varepsilon}}\left|D^{\varepsilon} u_{\varepsilon}\right|^{2}+\tilde{V}_{\varepsilon}\left|u_{\varepsilon}\right|^{2} \mathrm{~d} y=0,
$$

and thus, by elliptic estimates (see [29]), we obtain that

$$
\lim _{\varepsilon \rightarrow 0}\left\|u_{\varepsilon}\right\|_{L^{\infty}\left(\mathbb{R}^{N} \backslash\left(\mathcal{M}^{2 \beta}\right)_{\varepsilon}\right)}=0 .
$$

This gives the following decay estimate for $u_{\varepsilon}$ on $\mathbb{R}^{N} \backslash\left(\mathcal{M}^{2 \beta}\right)_{\varepsilon} \cup\left(Z^{\beta}\right)_{\varepsilon}$

$$
\left|u_{\varepsilon}(y)\right| \leq C \exp \left(-c \operatorname{dist}\left(y,\left(\mathcal{M}^{2 \beta}\right)_{\varepsilon} \cup\left(Z^{\beta}\right)_{\varepsilon}\right)\right)
$$

for some constants $C, c>0$. Indeed from (f1) and (3.47) we see that

$$
\lim _{\varepsilon \rightarrow 0}\left\|f\left(\left|u_{\varepsilon}\right|^{2}\right)\right\|_{L^{\infty}\left(\mathbb{R}^{N} \backslash\left(\mathcal{M}^{2 \beta}\right)_{\varepsilon} \cup\left(Z^{\beta}\right)_{\varepsilon}\right)}=0 .
$$

Also $\inf \left\{V_{\varepsilon}(y) \mid y \notin\left(\mathcal{M}^{2 \beta}\right)_{\varepsilon} \cup\left(Z^{\beta}\right)_{\varepsilon}\right\}>0$. Thus, we obtain the decay estimate (3.48) by applying standard comparison principles (see [39]) to (3.46).

If $Z \neq \emptyset$ we shall need, in addition, an estimate for $\left|u_{\varepsilon}\right|$ on $\left(Z^{2 \beta}\right)_{\varepsilon}$. Let $\left\{H^{i}\right\}_{i \in I}$ be the connected components of $\operatorname{int}\left(Z^{3 \delta}\right)$ for some index set $I$. Note that $Z \subset \bigcup_{i \in I} H^{i}$ and $Z$ is compact. Thus, the set $I$ is finite. For each $i \in I$, let $\left(\phi^{i}, \lambda_{1}^{i}\right)$ be a pair of first positive eigenfunction and eigenvalue of $-\Delta$ on $\left(H^{i}\right)_{\varepsilon}$ with Dirichlet boundary condition. From now we fix an arbitrary $i \in I$. By elliptic estimates [29], Theorem 9.20, and using the fact that $\left(Q_{\varepsilon}\left(u_{\varepsilon}\right)\right)$ is bounded we see that for some constant $C>0$

$$
\left\|u_{\varepsilon}\right\|_{L^{\infty}\left(\left(H^{i}\right)_{\varepsilon}\right)} \leq C \varepsilon^{3 / \mu} .
$$

Thus, from (f1) we have, for some $C>0$

$$
\left\|f\left(\left|u_{\varepsilon}\right|^{2}\right)\right\|_{L^{\infty}\left(\left(H^{i}\right)_{\varepsilon}\right)} \leq C \varepsilon^{3} .
$$

Denote $\phi_{\varepsilon}^{i}(y)=\phi^{i}(\varepsilon y)$. Then, for sufficiently small $\varepsilon>0$, we deduce that for $y \in \operatorname{int}\left(\left(H^{i}\right)_{\varepsilon}\right)$,

$$
\Delta \phi_{\varepsilon}^{i}(y)-V_{\varepsilon}(x) \phi_{\varepsilon}^{i}(y)+f\left(\left|u_{\varepsilon}(y)\right|^{2}\right) \phi_{\varepsilon}^{i}(y) \leq\left(C \varepsilon^{3}-\lambda_{1} \varepsilon^{2}\right) \phi_{\varepsilon}^{i} \leq 0 .
$$

Now, since $\operatorname{dist}\left(\partial\left(Z^{2 \beta}\right)_{\varepsilon},\left(Z^{\beta}\right)_{\varepsilon}\right)=\beta / \varepsilon$, we see from (3.48) that for some constants $C, c>0$,

$$
\left\|u_{\varepsilon}\right\|_{L^{\infty}\left(\partial\left(Z^{2 \beta}\right)_{\varepsilon}\right)} \leq C \exp (-c / \varepsilon) .
$$


We normalize $\phi^{i}$ requiring that

$$
\inf _{y \in\left(H^{i}\right)_{\varepsilon} \cap \partial\left(Z^{2 \delta}\right)_{\varepsilon}} \phi_{\varepsilon}^{i}(y)=C \exp (-c / \varepsilon)
$$

for the same $C, c>0$ as in (3.51). Then, we see that for some $\kappa>0$,

$$
\phi_{\varepsilon}^{i}(y) \leq \kappa C \exp (-c / \varepsilon), \quad y \in\left(H^{i}\right)_{\varepsilon} \cap\left(Z^{2 \beta}\right)_{\varepsilon} .
$$

Now we deduce, using (3.49)-(3.52) and [44], Theorem B.6, that for each $i \in I,\left|u_{\varepsilon}\right| \leq \phi_{\varepsilon}^{i}$ on $\left(H^{i}\right)_{\varepsilon} \cap\left(Z^{2 \beta}\right)_{\varepsilon}$. Therefore

$$
\left|u_{\varepsilon}(y)\right| \leq C \exp (-c / \varepsilon) \text { on }\left(Z^{2 \delta}\right)_{\varepsilon}
$$

for some $C, c>0$. Now (3.48) and (3.53) implies that $Q_{\varepsilon}\left(u_{\varepsilon}\right)=0$ for $\varepsilon>0$ sufficiently small and thus $u_{\varepsilon}$ satisfies (1.3). Now using Propositions 2.5 and 3.3, we readily deduce that the properties of $u_{\varepsilon}$ given in Theorem 1.1 hold. Here, in (1.7) we also use the fact, proved in Lemma 2.3, that any least energy solution of (2.11) has the form $\mathrm{e}^{i \tau} U$ where $U$ is a positive least energy solution of (2.7) and $\tau \in \mathbb{R}$.

Acknowledgements. The authors would like to thank the referee for many useful comments which help clarify the paper.

\section{REFERENCES}

[1] A. Ambrosetti, M. Badiale and S. Cingolani, Semiclassical states of nonlinear Schrödinger equations. Arch. Ration. Mech. Anal. 140 (1997) 285-300.

[2] A. Ambrosetti, A. Malchiodi and S. Secchi, Multiplicity results for some nonlinear Schrödinger equations with potentials. Arch. Ration. Mech. Anal. 159 (2001) 253-271.

[3] G. Arioli and A. Szulkin, A semilinear Schrödinger equations in the presence of a magnetic field. Arch. Ration. Mech. Anal. 170 (2003) 277-295.

[4] S. Barile, S. Cingolani and S. Secchi, Single-peaks for a magnetic Schrödinger equation with critical growth. Adv. Diff. Equations 11 (2006) 1135-1166.

[5] T. Bartsch, E.N. Dancer and S. Peng, On multi-bump semi-classical bound states of nonlinear Schrödinger equations with electromagnetic fields. Adv. Diff. Equations 11 (2006) 781-812.

[6] H. Berestycki and P.L. Lions, Nonlinear scalar field equation I. Arch. Ration. Mech. Anal. 82 (1983) 313-346.

[7] J. Byeon and L. Jeanjean, Standing waves for nonlinear Schrödinger equations with a general nonlinearity. Arch. Ration. Mech. Anal. 185 (2007) 185-200.

[8] J. Byeon and L. Jeanjean, Erratum: Standing waves for nonlinear Schrödinger equations with a general nonlinearity. Arch. Ration. Mech. Anal. DOI 10.1007/s00205-006-0019-3.

[9] J. Byeon and L. Jeanjean, Multi-peak standing waves for nonlinear Schrödinger equations with a general nonlinearity. Discrete Cont. Dyn. Systems 19 (2007) 255-269.

[10] J. Byeon and Z.-Q. Wang, Standing waves with critical frequency for nonlinear Schrödinger equations. Arch. Rat. Mech. Anal. 165 (2002) 295-316.

[11] J. Byeon and Z.-Q. Wang, Standing waves with critical frequency for nonlinear Schrödinger equations II. Calc. Var. Partial Differ. Equ. 18 (2003) 207-219.

[12] J. Byeon, L. Jeanjean and K. Tanaka, Standing waves for nonlinear Schrödinger equations with a general nonlinearity: one and two dimensional cases. Comm. Partial Diff. Eq. 33 (2008) 1113-1136.

[13] D. Cao and E.-S. Noussair, Multi-bump standing waves with a critical frequency for nonlinear Schrödinger equations. J. Diff. Eq. 203 (2004) 292-312.

[14] T. Cazenave, Semilinear Schrödinger equations, Courant Lecture Notes. AMS (2003).

[15] J. Chabrowski and A. Szulkin, On the Schrödinger equation involving a critical Sobolev exponent and magnetic field. Topol. Methods Nonlinear Anal. 25 (2005) 3-21.

[16] S. Cingolani, Semiclassical stationary states of nonlinear Schrödinger equations with an external magnetic field. J. Diff. Eq. 188 (2003) 52-79.

[17] S. Cingolani and M. Lazzo, Multiple positive solutions to nonlinear Schrödinger equations with competing potential functions. J. Diff. Eq. 160 (2000) 118-138.

[18] S. Cingolani and M. Nolasco, Multi-peaks periodic semiclassical states for a class of nonlinear Schrödinger equations. Proc. Royal Soc. Edinburgh 128 (1998) 1249-1260. 
[19] S. Cingolani and S. Secchi, Semiclassical limit for nonlinear Schrödinger equations with electromagnetic fields. J. Math. Anal. Appl. 275 (2002) 108-130.

[20] S. Cingolani and S. Secchi, Semiclassical states for NLS equations with magnetic potentials having polynomial growths. J. Math. Phys. 46 (2005) 1-19.

[21] M. Clapp, R. Iturriaga and A. Szulkin, Periodic solutions to a nonlinear Schrödinger equations with periodic magnetic field. Preprint.

[22] V. Coti-Zelati and P.H. Rabinowitz, Homoclinic orbits for second order Hamiltonian systems possessing superquadratic potentials. J. Amer. Math. Soc. 4 (1991) 693-727.

[23] V. Coti-Zelati and P.H. Rabinowitz, Homoclinic type solutions for a semilinear elliptic PDE on $\mathbb{R}^{N}$. Comm. Pure Appl. Math. 45 (1992) 1217-1269.

[24] M. Del Pino and P. Felmer, Local mountain passes for semilinear elliptic problems in unbounded domains. Calc. Var. Partial Differ. Equ. 4 (1996) 121-137.

[25] M. Del Pino and P. Felmer, Semi-classical states for nonlinear Schrödinger equations. J. Funct. Anal. 149 (1997) $245-265$.

[26] M. Del Pino and P. Felmer, Multi-peak bound states for nonlinear Schrödinger equations. Ann. Inst. H. Poincaré Anal. Non Linéaire 15 (1998) 127-149.

[27] M.J. Esteban and P.L. Lions, Stationary solutions of nonlinear Schrödinger equations with an external magnetic field, in PDE and Calculus of Variations, in honor of E. De Giorgi, Birkhäuser (1990).

[28] A. Floer and A. Weinstein, Nonspreading wave packets for the cubic Schrödinger equation with a bounded potential. J. Funct. Anal. 69 (1986) 397-408.

[29] D. Gilbarg and N.S. Trudinger, Elliptic Partial Differential Equations of Second Order, second edition, Grundlehren 224. Springer, Berlin, Heidelberg, New York and Tokyo (1983).

[30] C. Gui, Existence of multi-bump solutions for nonlinear Schrödinger equations. Comm. Partial Diff. Eq. 21 (1996) 787-820.

[31] H. Hajaiej and C.A. Stuart, On the variational approach to the stability of standing waves for the nonlinear Schrödinger equation. Advances Nonlinear Studies 4 (2004) 469-501.

[32] L. Jeanjean and K. Tanaka, A remark on least energy solutions in $\mathbb{R}^{N}$. Proc. Amer. Math. Soc. 131 (2003) $2399-2408$.

[33] L. Jeanjean and K. Tanaka, A note on a mountain pass characterization of least energy solutions. Advances Nonlinear Studies 3 (2003) 461-471.

[34] L. Jeanjean and K. Tanaka, Singularly perturbed elliptic problems with superlinear or asympotically linear nonlinearities. Calc. Var. Partial Diff. Equ. 21 (2004) 287-318.

[35] K. Kurata, Existence and semi-classical limit of the least energy solution to a nonlinear Schrödinger equation with electromagnetic fields. Nonlinear Anal. 41 (2000) 763-778.

[36] Y.Y. Li, On a singularly perturbed elliptic equation. Adv. Diff. Equations 2 (1997) 955-980.

[37] P.L. Lions, The concentration-compactness principle in the calculus of variations. The locally compact case. Part II. Ann. Inst. H. Poincaré Anal. Non Linéaire 1 (1984) 223-283.

[38] Y.G. Oh, Existence of semiclassical bound states of nonlinear Schrödinger equations. Comm. Partial Diff. Eq. 13 (1988) 1499-1519.

[39] M.H. Protter and H.F. Weinberger, Maximum Principles in Differential Equations. Springer-Verlag, New York, Berlin, Heidelberg and Tokyo (1984).

[40] P. Rabinowitz, On a class of nonlinear Schrödinger equations. Z. Angew. Math. Phys. 43 (1992) $270-291$.

[41] M. Reed and B. Simon, Methods of Modern Mathematical Physics, Vol. II. Academic press, New York (1972).

[42] S. Secchi and M. Squassina, On the location of spikes for the Schrödinger equations with electromagnetic field. Commun. Contemp. Math. 7 (2005) 251-268.

[43] W. Strauss, Existence of solitary waves in higher dimensions. Comm. Math. Phys. 55 (1977) $149-162$.

[44] M. Struwe, Variational Methods, Application to Nonlinear Partial Differential Equations and Hamiltonian Systems. SpringerVerlag (1990).

[45] X. Wang and B. Zeng, On concentration of positive bound states of nonlinear Schrödinger equation with competing potential functions. SIAM J. Math. Anal. 28 (1997) 633-655. 